University of Tennessee Health Science Center

UTHSC Digital Commons

\title{
$5-2009$
}

\section{Feasibility Study of Liquid-Based Cytology for Post-Treatment Surveillance of Patients with Vulvar Intraepithelial Neoplasia}

\author{
Wendy Likes \\ University of Tennessee Health Science Center
}

Follow this and additional works at: https://dc.uthsc.edu/dissertations

Part of the Diagnosis Commons, Female Urogenital Diseases and Pregnancy Complications Commons, and the Other Analytical, Diagnostic and Therapeutic Techniques and Equipment Commons

\section{Recommended Citation}

Likes, Wendy , "Feasibility Study of Liquid-Based Cytology for Post-Treatment Surveillance of Patients with Vulvar Intraepithelial Neoplasia" (2009). Theses and Dissertations (ETD). Paper 158. http://dx.doi.org/ 10.21007/etd.cghs.2009.0183. 


\title{
Feasibility Study of Liquid-Based Cytology for Post-Treatment Surveillance of Patients with Vulvar Intraepithelial Neoplasia
}

\begin{abstract}
Introduction: Colposcopy and tissue biopsy remain the gold standard for diagnosing vulvar intraepithelial neoplasia (VIN). Vulvar colposcopy is fairly nonspecific. As a result, many women undergo unwarranted painful biopsies. Vulvar cytology, which is relatively painless, inexpensive and allows a larger area to be sampled, may likely reduce false negative diagnoses. However, previous cytological studies that used conventional methodologies were largely unsuccessful in diagnosing VIN. In this study, liquid based cytology and HPV typing by PCR have been assessed as possible alternatives to biopsy for follow-up surveillance of women treated for VIN.

Methods: Women with a history of VIN and a control group were recruited from a colposcopy clinic. Clinically suspicious lesions and normal vulvar tissue were vigorously brushed and the sample collected in PreservCyt ${ }^{\circledR}$ fluid for cytologic examination and for HPV-typing with a multiplex PCR assay, using primers designated PGMY09/11. Samples $(\mathrm{N}=82)$ were obtained from 52 lesions clinically suspicious for VIN, 15 controls from the same women in areas of the vulva with no clinical abnormality, and 15 controls from women with no current clinical evidence or past history of VIN. Concurrent tissue biopsies were obtained, immediately after brushing, from the 52 clinically suspicious VIN lesions. A single pathologist read and interpreted the biopsy samples. To ensure unbiased testing, cytology and HPV analyses were performed at separate independent laboratories by professionals blinded to clinical findings and biopsy results. Cytology was coded as negative, ASCUS, VIN I, VIN 2, or VIN 3. Specificity, sensitivity, and predictive values of cytology and HPV for VIN were calculated. Fisher's exact was used to determine associations between HPV and cytology. Logistic regression was done to determine if cytology or HPV predicted tissue biopsy results.
\end{abstract}

Results: Vulvar samples ( $\mathrm{N}=82)$ were collected from 48 women aged 19-65 who participated in this study. Histology results of the 52 lesions clinically suspected as VIN were reported as follows: VIN I $(n=33)$, VIN $2 / 3(n=13)$, benign $(n=4)$, contact dermatitis and condyloma $(n=1$ each). Ninety percent of the vulvar samples were adequate for cytologic evaluation, but only $72 \%$ of samples had adequate cellularity for HPV testing. Sensitivity, specificity and positive predictive value (PPV) of vulvar cytology for recurrent VIN were $95 \%, 15 \%$ and $65 \%$ respectively. PCR for HPV, when independently correlated with histology, had, $62 \%$ sensitivity, $85 \%$ specificity and $89 \%$ PPV for VIN. No significant associations were found between cytology and HPV $(p=0.3559)$. Neither cytology nor HPV predicted pathological diagnosis of VIN.

Conclusion: By vigorous brushing, it is possible to obtain an adequate cellular sample from the vulva for cytologic and/or molecular evaluation for HPV.The specificity for VIN at cytology was not satisfactory for use as a clinical alternative to biopsy to detect recurrent VIN. This may be due to the small sample size, difficulty in accurately grading vulvar dysplasia at cytology, and possible differences in cytomorphologic criteria for diagnosing dysplasia in the vulva as compared to the cervix. This study will be further refined with the development of more reproducible consensus criteria for cytologic evaluation of VIN. A larger number of participants could shed more light on the significance of cytology with or without HPV testing as an alternative to tissue biopsy for follow up of patients with VIN.

Document Type

Dissertation

Degree Name

Doctor of Philosophy (PhD) 


\section{Program}

Nursing

Research Advisor

Donna Hathaway, PhD

\section{Keywords}

cancer; cytology; HPV; precancer; screening; Vulva

\section{Subject Categories}

Diagnosis | Diseases | Female Urogenital Diseases and Pregnancy Complications | Medicine and Health Sciences | Other Analytical, Diagnostic and Therapeutic Techniques and Equipment 


\title{
FEASIBILITY STUDY OF LIQUID-BASED CYTOLOGY FOR POST- TREATMENT SURVEILLANCE OF PATIENTS WITH VULVAR INTRAEPITHELIAL NEOPLASIA
}

\author{
A Dissertation \\ Presented for \\ The Graduate Studies Council \\ The University of Tennessee \\ Health Science Center
}

\author{
In Partial Fulfillment \\ Of the Requirements for the Degree \\ Doctor of Philosophy \\ From The University of Tennessee
}

By

Wendy Likes

May 2009 
Copyright (C) 2009 by Wendy Likes

All rights reserved 


\section{DEDICATION}

This dissertation is dedicated to my husband, Billy Likes, and children,

Jacob and Joshua Likes, for their support and encouragement throughout my academic endeavors. 


\section{ACKNOWLEDGEMENTS}

I would like to thank Dr. Donna Hathaway for her assistance in this academic milestone. I would also like to sincerely thank my committee members, Drs. Patricia Cowan, Barbara Benstein, Jim Wan, and Nadeem Zafar, for their valuable input and time. I would also like to extend my gratitude to my husband, children, parents, in-laws, and colleagues for their patience and support.

Funding for this study was provided by the University of Tennessee Health Science Center General Clinical Research Center. This research would not have been possible without their support. 


\begin{abstract}
Introduction: Colposcopy and tissue biopsy remain the gold standard for diagnosing vulvar intraepithelial neoplasia (VIN). Vulvar colposcopy is fairly nonspecific. As a result, many women undergo unwarranted painful biopsies. Vulvar cytology, which is relatively painless, inexpensive and allows a larger area to be sampled, may likely reduce false negative diagnoses. However, previous cytological studies that used conventional methodologies were largely unsuccessful in diagnosing VIN. In this study, liquid based cytology and HPV typing by PCR have been assessed as possible alternatives to biopsy for follow-up surveillance of women treated for VIN.
\end{abstract}

Methods: Women with a history of VIN and a control group were recruited from a colposcopy clinic. Clinically suspicious lesions and normal vulvar tissue were vigorously brushed and the sample collected in PreservCyt ${ }^{\circledR}$ fluid for cytologic examination and for HPV-typing with a multiplex PCR assay, using primers designated PGMY09/11. Samples $(\mathrm{N}=82)$ were obtained from 52 lesions clinically suspicious for VIN, 15 controls from the same women in areas of the vulva with no clinical abnormality, and 15 controls from women with no current clinical evidence or past history of VIN. Concurrent tissue biopsies were obtained, immediately after brushing, from the 52 clinically suspicious VIN lesions. A single pathologist read and interpreted the biopsy samples. To ensure unbiased testing, cytology and HPV analyses were performed at separate independent laboratories by professionals blinded to clinical findings and biopsy results. Cytology was coded as negative, ASCUS, VIN I, VIN 2, or VIN 3. Specificity, sensitivity, and predictive values of cytology and HPV for VIN were calculated. Fisher's exact was used to determine associations between HPV and cytology. Logistic regression was done to determine if cytology or HPV predicted tissue biopsy results.

Results: Vulvar samples $(\mathrm{N}=82)$ were collected from 48 women aged 19-65 who participated in this study. Histology results of the 52 lesions clinically suspected as VIN were reported as follows: VIN I $(n=33)$, VIN $2 / 3(n=13)$, benign $(n=4)$, contact dermatitis and condyloma $(n=1$ each). Ninety percent of the vulvar samples were adequate for cytologic evaluation, but only $72 \%$ of samples had adequate cellularity for HPV testing. Sensitivity, specificity and positive predictive value (PPV) of vulvar cytology for recurrent VIN were 95\%, 15\% and 65\% respectively. PCR for HPV, when independently correlated with histology, had, 62\% sensitivity, $85 \%$ specificity and $89 \%$ PPV for VIN. No significant associations were found between cytology and HPV ( $p=0.3559)$. Neither cytology nor HPV predicted pathological diagnosis of VIN.

Conclusion: By vigorous brushing, it is possible to obtain an adequate cellular sample from the vulva for cytologic and/or molecular evaluation for HPV. The specificity for VIN at cytology was not satisfactory for use as a clinical alternative to biopsy to detect recurrent VIN. This may be due to the small sample size, difficulty in accurately grading vulvar dysplasia at cytology, and possible differences in cytomorphologic criteria for diagnosing dysplasia in the vulva as compared to the cervix. This study will be further refined with the development of more reproducible consensus criteria for cytologic 
evaluation of VIN. A larger number of participants could shed more light on the significance of cytology with or without HPV testing as an alternative to tissue biopsy for follow up of patients with VIN. 


\section{TABLE OF CONTENTS}

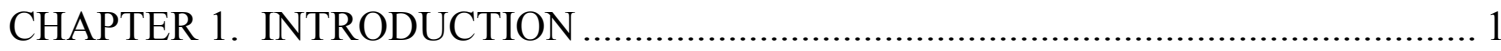

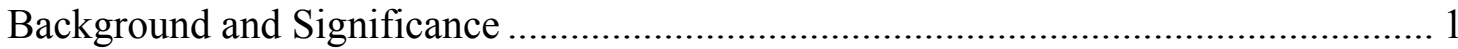

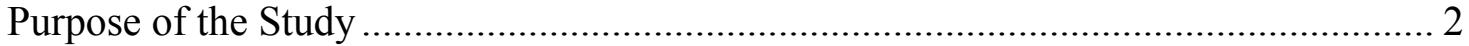

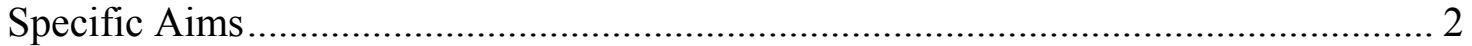

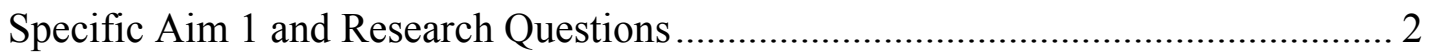

Specific Aim 2 and Research Questions ............................................................ 3

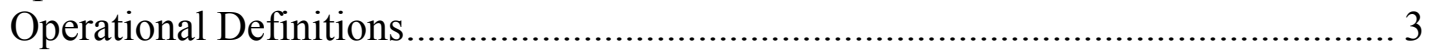

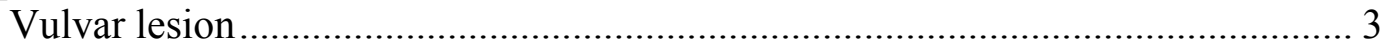

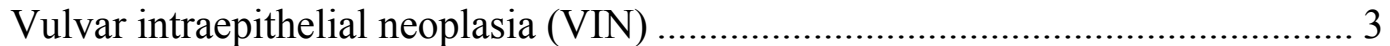

Human papillomavirus (HPV) ................................................................. 3

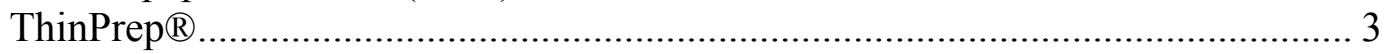

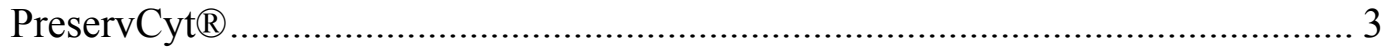

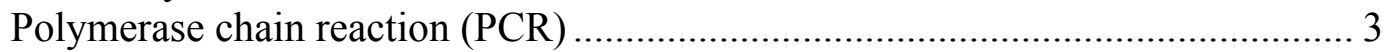

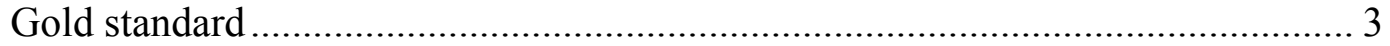

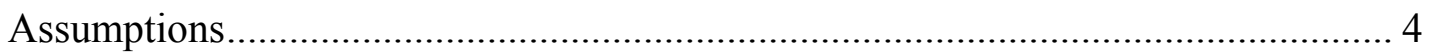

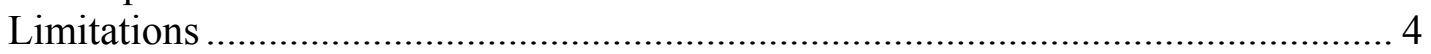

Conceptual Model: Linking HPV Changes to the Clinical Pathway in VIN.............. 4

Human Papillomavirus................................................................................. 4

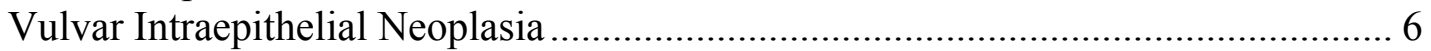

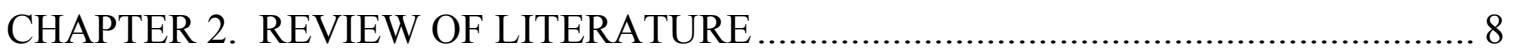

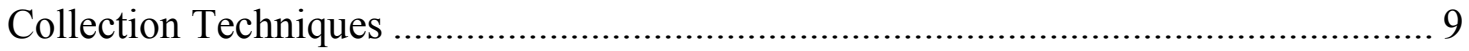

Vulvar Cytology and Histology .............................................................................. 9

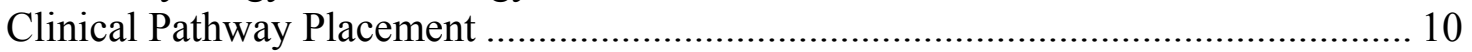

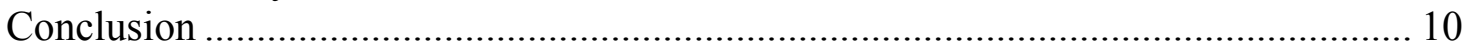

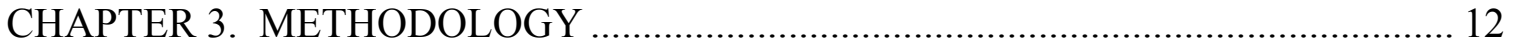

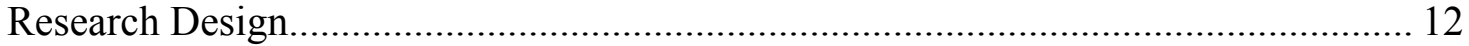

Specific Aim 1 and Research Questions .............................................................. 12

Specific Aim 2 and Research Questions ......................................................... 12

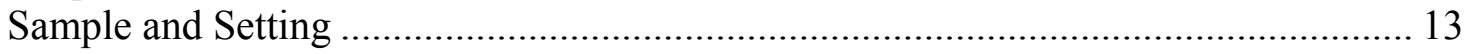

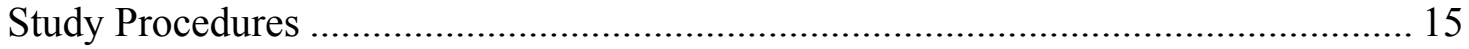

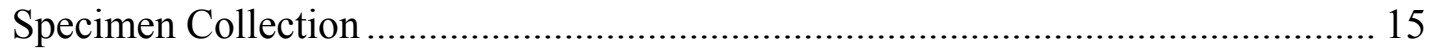

Cytology and Human Papillomavirus ................................................................ 16

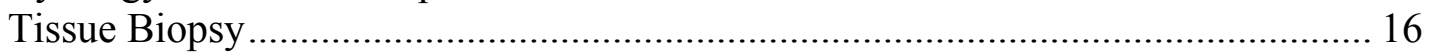

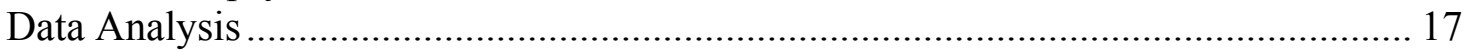

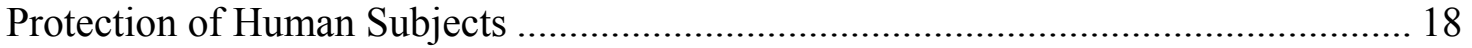

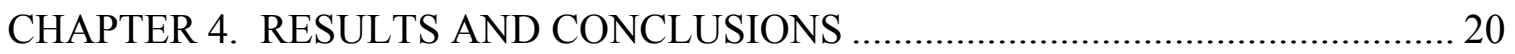

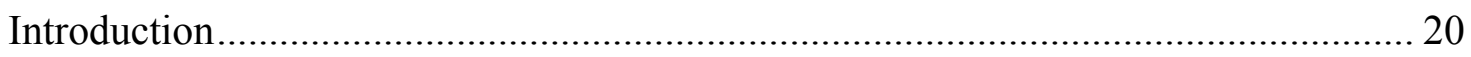

Specific Aim 1 and Research Questions .............................................................. 20

Specific Aim 2 and Research Questions .............................................................. 21

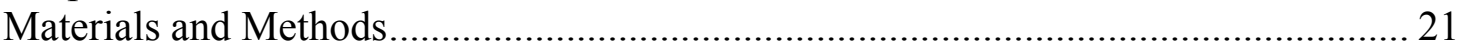




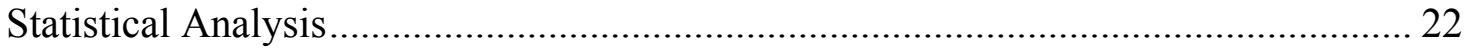

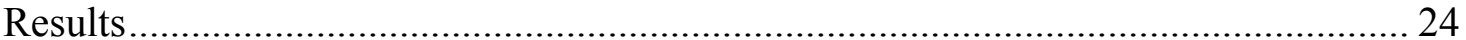

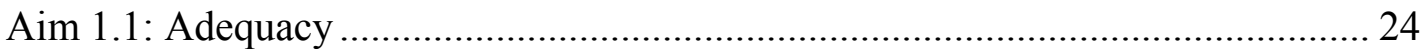

Aims 1.2-1.4 and Aim 2.3: Sensitivity, Specificity and Predictive Value................ 24

Aim 2.1 and 2.2: Association of Cytology to HPV PCR / Pathology ....................... 31

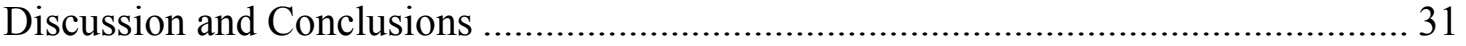

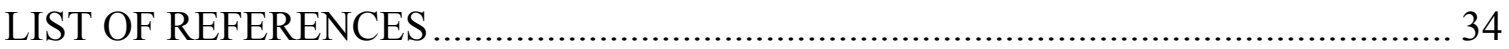

APPENDIX A. INSTITUTIONAL REVIEW BOARD APPROVAL .......................... 38

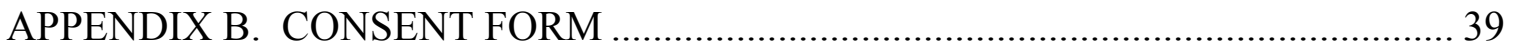

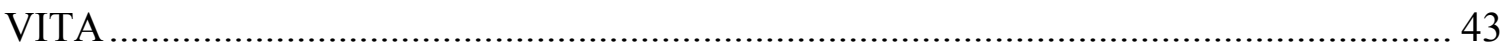




\section{LIST OF TABLES}

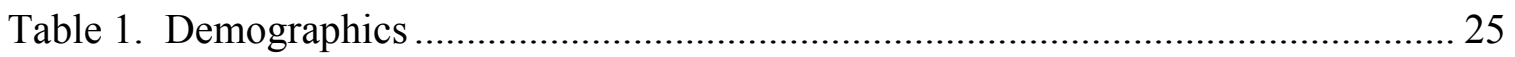

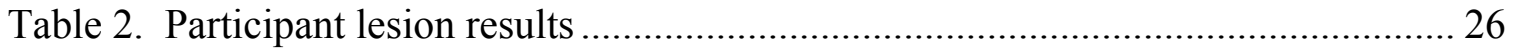

Table 3. Comparison of cytology test results to biopsy results .................................... 29

Table 4. Comparison of HPV test results to biopsy results ....................................... 30

Table 5. Comparison of cytology/HPV reflux test results to biopsy results................... 33 


\section{CHAPTER 1. INTRODUCTION}

Vulvar cancer is a rare disease traditionally found in older women with a peak incidence in the $6^{\text {th }}$ and $7^{\text {th }}$ decade (Sturgeon, Brinton, Devesa, \& Kurman, 1992). Over the last ten to twenty years, there has been a significant increase in vulvar intraepithelial neoplasia (VIN), a family of lesions which are precursors to vulvar carcinoma. VIN can be due to human papillomavirus (HPV), called undifferentiated VIN, or due to an epithelial disorder, known as differentiated VIN. Undifferentiated VIN, related to HPV is typically observed in younger women, defined in most literature as less than the age of 50. The increase in VIN has been most dramatic among younger women and is attributed to several factors, most noticeably increased exposure to HPV (Jones, Baranyai, \& Stables, 1997; Joura, Losch, Haider-Angeler, Breitenecker, \& Leodolter, 2000). VIN also has a high rate of recurrence. The current standard of care therefore dictates close followup with colposcopic examinations and periodic biopsies to rule out recurrence (Sturgeon et al., 1992). However, even with the aid of a colposcope, it is difficult to distinguish between benign lesions resulting from infections, various skin conditions or scar tissue from neoplastic or dysplastic lesions (Likes, 2009). Therefore, all lesions are typically subjected to tissue evaluation through biopsies. Tissue biopsies are invasive and painful and women with VIN describe them as stressful and anxiety provoking (Likes, Russell, \& Tillmanns, 2008).

A new method for monitoring women with vulvar lesions is needed that would be less painful and anxiety-laden and thereby enhance patient compliance, follow-up, and successful treatment. The Papanicolaou test (Pap smear), which has been highly successful in the triage of patients for cervical cancer, has failed to show similar success in pilot studies triaging vulvar neoplasia and dysplasia. These studies employed conventional sampling techniques and cytologic sample processing methodology. New liquid based cytologic techniques and ancillary molecular markers have shown increased efficacy in detecting cervical neoplasia and therefore could potentially allow efficient and accurate evaluation of VIN without the pain and anxiety associated with tissue biopsy.

\section{Background and Significance}

The Pap test, which involves collection of cells from the mucosal surface of the cervix for microscopic study, has been widely successful in detecting cervical malignancy and premalignant conditions. Previous attempts to use cytologic methods to identify VIN have been largely unsuccessful due to problems with suboptimal sample collection and drying of the cellular material, thus interfering with optimal diagnostic evaluation (Dennerstein, 1968, 1988; Nauth, 1986; Nauth \& Boger, 1982). These reports also cite poor correlation between vulvar cytology and biopsy results. However, these studies did not employ modern collection devices or current and more optimal liquid based cytologic processing methods. Even in the most recent study employing vulvar cytology (Bae-Jump, Bauer, \& Van Le, 2007), only 6 of the 50 patients were evaluated using the ThinPrep ${ }^{\circledR}$ technique (Cytyc Corporation Marlborough, MA) and the specimens were collected using the spatula sampling device rather than the more modern 
brush or broom-type collection devices. In addition, this study utilized a retrospective chart review without established consistency in sampling of the lesion. Cytology, specifically cervical cytology, is a technique familiar to most health care practitioners. Vulvar cytology can be performed using the same equipment as Pap smears. After properly defining the technique and the mechanics of the procedure, vulvar cytology would require a less sophisticated skill set than tissue biopsy and would be more readily available to practitioners. It can be hypothesized, that using vulvar cytology would allow for a wider screening of abnormal vulvar lesions within a faster time frame than if the health care provider referred the patient to a specialist for tissue biopsy. Therefore, preinvasive lesions could be found with greater ease before progression to cancer occurs resulting in decreased morbidity and mortality from vulvar cancer.

\section{Purpose of the Study}

The standard diagnostic procedure for VIN remains visualization and biopsy. However innovative and less invasive sampling and evaluation techniques could be introduced, which are likely to improve patient compliance without compromising diagnostic accuracy. The use of liquid-based techniques allows for cytologic interpretations to be made from these vulvar lesions without actually obtaining tissue through puncture. Therefore, the purpose of this study was to determine the feasibility of developing a protocol for cytologic sampling of vulvar lesions and to evaluate the efficacy of vulvar cytology in conjunction with Human Papillomavirus (HPV) testing as a valid method for following women treated for vulvar intraepithelial neoplasia (VIN).

\section{Specific Aims}

In contrast to previous studies, this feasibility study utilized a nylon brush and saline to moisten and exfoliate cellular material from the lesions. The cellular material was then immersed into PreservCyt ${ }^{\circledR}$ solution for ThinPrep processing, which is believed to enhance the adequacy of specimens and be more effective in the identification of cervical cancer precursors than other methods. A second sample was collected for HPV typing using the polymerase chain reaction (PCR) method. The specific aims and research questions of this study were:

\section{Specific Aim 1 and Research Questions}

To determine the feasibility of a protocol for cytologic sampling of vulvar lesions.

1.1 Does the vulvar sampling technique provide adequate cellularity for cytologic examination?

1.2 What is the sensitivity of vulvar cytology for detecting VIN?

1.3 What is the specificity of vulvar cytology for detecting VIN?

1.4 What are the negative and positive predictive values of vulvar cytology for detecting VIN? 


\section{Specific Aim 2 and Research Questions}

To evaluate the efficacy of vulvar cytology in conjunction with Human Papillomavirus (HPV) testing as a valid method for following women treated for vulvar intraepithelial neoplasia.

2.1 What is the relationship between vulvar cytology results and HPV results?

2.2 Can HPV testing in combination with vulvar cytology, while controlling for age, be utilized as a triage tool for tissue biopsy results?

2.3 What is the accuracy of PCR analysis for HPV as a predictor of vulvar intraepithelial neoplasia?

2.4 If an association is found between cytology/HPV and tissue biopsy results, then determine if cytology in conjunction with HPV testing predict the biopsy outcome?

\section{Operational Definitions}

Vulvar lesion - A clinically apparent vulvar lesion that may be flat or raised, discolored, and/or ulcerated OR an area of acetowhite change after application of acetic acid.

Vulvar intraepithelial neoplasia (VIN)—A precancerous condition of the vulva diagnosed through vulvar biposy.

Human papillomavirus (HPV) - A virus that interferes with the cell's ability to prevent excessive growth and may potentially lead to precancerous and cancerous conditions. HPV is detected through polymerase chain reaction (PCR).

ThinPrep ${ }^{\circledR}$-A liquid based process for cytologic interpretation.

PreservCyt ${ }^{\circledR}$ - A specimen collection fluid for preservation of cytologic material for up to 6 weeks at $15-30^{\circ} \mathrm{C}$.

Polymerase chain reaction (PCR) - A key technique in molecular genetics that permits the analysis of any short sequence of DNA (or RNA) without having to clone it. PCR is used to reproduce (amplify) selected sections of DNA.

Gold standard-For comparison of the diagnostic tests under evaluation was tissue biopsy or in absence of tissue biopsy, HPV typing. 


\section{Assumptions}

The framework of this study is grounded on the following assumptions.

- The "gold standard" for diagnosis of VIN is vulvar biopsy.

- Participants who are HR HPV negative do not have VIN.

- If the "gold standard" is not available, then HPV testing is an appropriate substitute to determine that a woman would not have VIN

- Participants who are healthy controls, and have a negative cytology, do not have VIN.

- Factors influencing the method used for diagnosis include: patient discomfort, risks of the procedure, cost, accuracy, distress, and ease of collection.

\section{Limitations}

The following limitations were considered during the conduct and in interpreting the outcomes of this study: Tissue biopsy was not collected on those who were healthy controls.

\section{Conceptual Model: Linking HPV Changes to the Clinical Pathway in VIN}

The conceptual model was constructed using relevant published literature and knowledge based on clinical experience in the study population (Figure 1). The model depicts the cellular changes from HPV, the development of VIN, and the detection of these changes. The details of this model are described in the following sections.

\section{Human Papillomavirus}

Papillomavirus are a diverse group of DNA-based viruses that typically affect the epithelium in humans. Over 100 types of HPV have been identified with each infecting specific epithelial sites (Longworth \& Laimins, 2004). There are two main HPV genere, alpha and beta. Beta papillomavirus affects cutaneous areas whereas alpha papillomavirus infects genital and mucosal surfaces. HPV's are broadly categorized into high risk (HRHPV), intermediate risk (IR HPV), and low risk (LR HPV) types. HR HPV are the types most commonly associated with the development of cancer (Kagie et al., 1997). LR HPV and IR HPV are rarely implicated in cancer development and more commonly associated with the development of warts. HR HPV infection is commonly observed and although it has the potential to cause cancer, the association of HR HPV infection with cancer is not inevitable.

HPV infection is a non-lytic infection and thereby can escape recognition by the host's immune system (Kanodia, Fahey, \& Kast, 2007). HPV is believed to enter the host through micro-abrasions on the epithelial surface. The HPV virion establishes itself in 


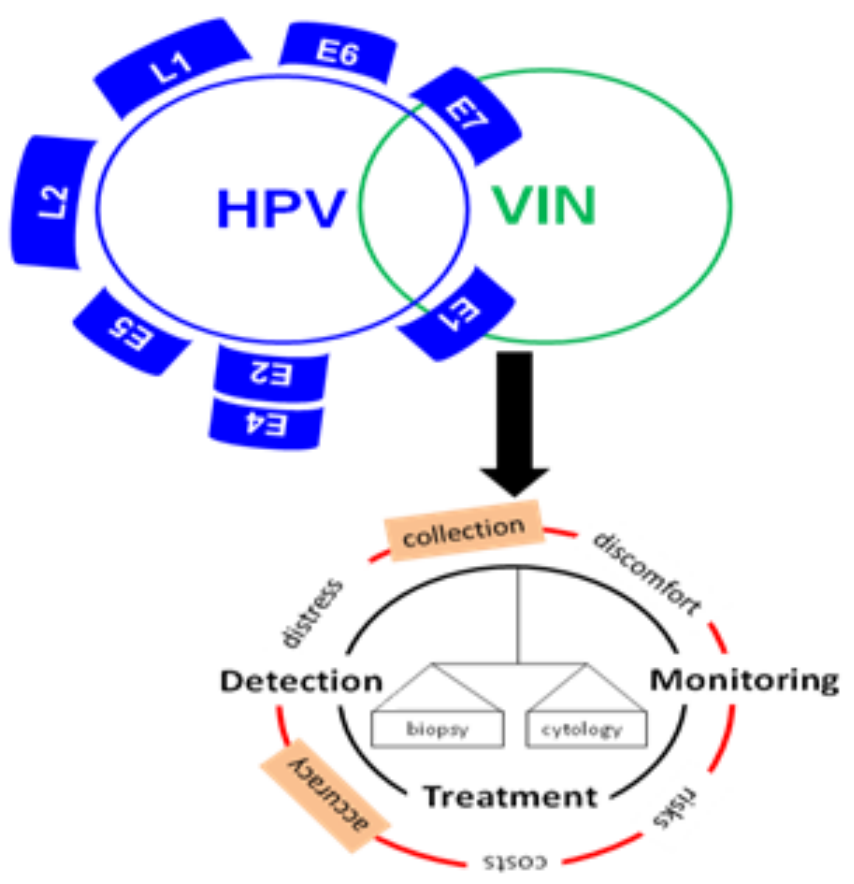

Figure 1. Conceptual model 
the basal layer of the epithelium and enters the epithelial cells through endocytosis. The HPV genome is comprised of 6 early proteins (E1, E2, E4, E5, E6, E7) and two late proteins (L1 \& L2). E6 and E7, the first genes to be expressed, are the major transforming genes and cause an increase in cellular division (Likes \& Itano, 2003; Longworth \& Laimins, 2004). As these cells divide, they carry the HPV DNA with them. E1 possesses DNA-dependent ATPase and ATP dependent helicase activity which assists in unwinding of the host DNA. E1 and E2 are responsible for viral replication.

This replication process appears to occur during the S-phase of the cell cycle. E4 and E5 encode a protein required for the final stage of replication. The viral oncogenes, E6 and E7 are thought to modify the host's cell cycle to continue viral genome replication by driving it into the S-phase. The E5 protein is capable of inactivating the tumor suppressor gene p21 while E6 combines to the tumor suppressor gene p53 and targets it for destruction. In addition, E7 binds to the tumor suppressor gene Rb1 or p107 and inactivates it leading to deregulated growth control in the epithelial cells. With the combined action of these proteins, the restraint of the cell-cycle progression is lost and normal terminal differentiation does not occur. The cell does not respond to the cellular DNA damage and secondary mutations are allowed to go unchecked. The relative thickness of epithelium increases as the grade of neoplasia increases and the extent of differentiation decreases (Doorbar, 2006). L1 and L2 produces viral like particles and are expressed in the differentiation cycle of the keratinocytes and other HR HPV affected epithelial cells. In skin, these virus- like particles are shed with the dead skin cells. The HPV life cycle is represented in the model with the circular structure that depicts the various proteins as described above.

\section{Vulvar Intraepithelial Neoplasia}

Vulvar Intraepithelial Neoplasia includes two types of lesions, differentiated and usual type. Differentiated VIN is not related to HPV and typically arises in a background of lichen sclerosis or squamous cell hyperplasia. This type of VIN usually occurs in women over the age of 50 (Stroup, Harlan, \& Trimble, 2008). Differentiated VIN has been found to chronically progress from oxidative damage to genetic instability, atypia, and finally cancer (Kagie et al., 1997; Lin et al., 1998). This type of VIN represents approximately $20 \%$ of all VIN cases and will not be represented in this study. Thus, the overlap of VIN and HPV in the conceptual model for this study is not complete since not all VIN's are caused by HPV.

This study will focus on usual type VIN. Usual type VIN is typically related to HR HPV types, particularly 16, 18, 31, and 33 (Vinokurova et al., 2005). VIN usual type, is associated with warty and basaloid squamous carcinomas which are HPV related tumors (Stroup et al., 2008).

The gold standard for the diagnosis of VIN is tissue biopsy. However, historically vulvar cytology was used as a triage tool prior to biopsy. However, this practice has been largely abandoned. The current study re-evaluates the use of vulvar cytology. In the 
conceptual model, biopsy and cytology are depicted as scales in that this study will determine if cytology is equivalent to biopsy or appropriate as a triage tool prior to biopsy. These tests may be used for detection, monitoring, and for the evaluation of treatment in women with VIN. This is depicted as cyclic since VIN has a high recurrence rate and therefore, the diagnostic method chosen will continually be utilized for the detection of recurrent disease, monitoring of disease, and evaluating treatment effectiveness.

The outer circle in the model depicts the assumed influences on the diagnostic method chosen. A hierarchical classification system for the clinical endpoints of diagnostic technology assessment has been developed. Pearl developed a system that utilizes 6 categories for investigation of outcomes: technical efficacy, diagnostic accuracy efficacy, diagnostic thinking efficacy, therapeutic efficacy, clinical outcome efficacy, and societal efficacy (Pearl, 1999) This study focuses on the first two categories of this hierarchical model; technical efficacy and diagnostic accuracy efficacy. These two categories are depicted in the outer ring in orange highlighted boxes. Technical efficacy will be evaluated through feasibility of the method by determining if adequate numbers of cells are obtained for evaluation. Diagnostic accuracy efficacy will be evaluated by the sensitivity, specificity, and predictive values of vulvar cytology. Additional factors that influence the testing method include: discomfort, risks, costs, distress, and ease of collection. Vulvar cytology is assumed to cause less discomfort and fewer risks since it is not an invasive procedure. This is felt to lead to less distress. In previous focus groups, women with VIN identified biopsies as painful and anxiety provoking (Likes et al., 2008). If cytology is found to be an appropriate method for clinical management of these women, this aspect of the model should be evaluated. Vulvar colposcopy with tissue biopsy costs approximately $\$ 350.00$ whereas cytology is approximately $\$ 35.00$. Further studies will need to be conducted on the cost-benefit of using cytology. In addition, vulvar cytology requires less technical skill and equipment to perform and should be more readily available for use by healthcare providers, thus making it a feasible method for clinical evaluation. These additional influences in the model will need to be analyzed if the data from this study is in support of using cytology. 


\section{CHAPTER 2. REVIEW OF LITERATURE}

Several studies have reported that vulvar cancer and its precursor, vulvar intraepithelial neoplasia (VIN) are increasing (Joura et al., 2000; Judson, Habermann, Baxter, Durham, \& Virnig, 2006; Maclean, 2006; Sturgeon et al., 1992). Over the last several decades, the incidence of VIN has doubled in all age groups and tripled in those under the age of 50 (Joura et al., 2000; Sturgeon et al., 1992). In addition, it was found recently that the incidence of vulvar cancer increased by $20 \%$ in the United States between 1973 and 2000 (Judson et al., 2006). This increase in VIN and vulvar cancer is concerning, however more alarming is the doubling of cases of vulvar cancer in women under the age of 50 years (Maclean, 2006). This increase is postulated to be due to the increased rate of human papillomavirus (HPV), a sexually transmitted virus. A vaccine has been developed to cover HPV types $6,11,16$, and 18 . The advent of the vaccine holds much promise for the prevention of this disease; however studies have yielded variable results on the types of HPV implicated in VIN and vulvar cancer. One study found $84 \%$ of high grade VIN (HGVIN) was due to HPV types 16/18. In addition, of the $65 \%$ of vulvar cancers related to HPV, approximately 55\% were due to HPV 16/18 (Insinga, Dasbach, Elbasha, Puig, \& Reynales-Shigematsu, 2007). Skapa found similar results, but also found HPV 33 and HPV 45 can be implicated in approximately $27 \%$ of vulvar cancers and $20 \%$ of VIN lesions (Skapa et al., 2007). Screening measures, as well as preventive measures, are paramount in this disease process as rates of VIN and vulvar cancer are increasing.

The gold standard for diagnosis of vulvar cancer and VIN is tissue biopsy of the vulvar lesion. Women with VIN present with a high recurrence rate noted to range from 18-52\% (Hillemanns, Wang, Staehle, Michels, \& Dannecker, 2006; Jones, Rowan, \& Stewart, 2005; Thuis, Campion, Fox, \& Hacker, 2000). Women with VIN are typically followed every 3 to 6 months with vulva colposcopy. If an abnormal lesion is found, the gold standard is tissue biopsy. Vulvar colposcopy is fairly nonspecific with many nonneoplastic disorders presenting with aceto-white changes that may mimic VIN. Many women undergo vulvar biopsy for conditions that may not warrant tissue biopsy. Historically, vulvar cytology would have been used as a triage tool; however this method has been abandoned for multiple reasons as previously stated. Vulvar cytology remains appealing since it can sample a larger area rather than one $3-4 \mathrm{~mm}$ area taken by a punch biopsy and therefore may result in less false negatives. Vulvar cytology is also less expensive, less invasive and may spare some women from the need of a painful tissue biopsy. The main issues that need to be addressed are: what is the most appropriate collection technique to obtain an adequate sample, how does vulvar cytology compare to the gold standard, and where in the clinical pathway is vulvar cytology most appropriate? This paper will review the literature in regard to these questions. However the literature is sparse and most of it is outdated. In addition, newer technologies, such as liquid-based cytology have been developed since most of these studies. Liquid-based cytology offers the ability to collect a sample with less obscuring material. In addition, air-drying that occurred with previous slide-based technology does not occur in liquid-based cytology. 


\section{Collection Techniques}

The first published account on vulvar cytology was reported in 1950 but focused on the classification of vulvar smears (Carter, 1950). Dennerstein published the first account of a vulvar cytology collection technique eighteen years later (Dennerstein, 1968). He noted that in order to adequately sample the vulva, the tissue should be scraped with a scalpel after soaking the area with a saline swab. Since then, much debate has occurred over the collection technique that would yield adequate cells for examination. In 1988, Dennerstein compared three different collection techniques (Dennerstein, 1988); a scalpel (as he advocated earlier), a swab stick, and a spatula. The scalpel method was found to be the most diagnostically useful. Ayala and Ayal used the scalpel technique in their study on vulvar terminology with a reported $1 \%$ unsatisfactory rate (Ayala et al., 1976). The most recent published study on vulvar cytology also utilized the scalpel method with fixation to a slide and also included 6 cases using liquid-based cytology (Bae-Jump et al., 2007). The authors did not specifically report any unsatisfactory sample cases. In 2001, Levine and colleagues utilized a technique with a 2-cm nylon brush rotated over the vulva 20 times and then placed in tissue culture fluid and processed using a cytospin technique (Levine et al., 2001). All collected specimens were determined to have adequate cellularity. Maclean (Maclean, 2006)also used the nylon brush technique but reported a $14 \%$ unsatisfactory rate due to low cellularity. Nauth and colleagues published a series of papers on vulvar cytology using a pre-moistened saline swab as the collection device (Nauth, 1981, 1986; Nauth \& Boger, 1982; Nauth, Neumann, \& Feilen, 1987). This technique was successful throughout several studies. Kashimura et al. obtained cytological samples by vigorously scraping the edge of a slide against the tissue after application of saline solution to avoid drying (Kashimura, Matsuura, Kawagoe, Toki, \& Sugihara, 1993). The authors did not indicate any problems due to lack of cellularity. With the development of liquid-based cytology, a nylon brush maybe the most appropriate collection technique, but research is needed to determine its adequacy with this methodology.

\section{Vulvar Cytology and Histology}

Six studies were found that addressed the correlation of vulvar cytology to histology. Comparison across these studies are difficult due to the different analyses used, however, most studies reported high correlation up to 91\% (Levine et al., 2001; Maclean, 2006; Nauth, 1986). Levine and associates reported 100\% sensitivity with no false negatives among a sample of 23 women; however, in a larger study $(\mathrm{N}=123)$ by Nauth, up to $30 \%$ of benign conditions were reported as abnormal. In 56 samples, JimenezAyala and Jimenez-Ayala also reported no false negatives with a sensitivity of $96.7 \%$ for benign conditions and 98.12 for malignant conditions (Jimenez-Ayala \& Jimenez-Ayala, 2002). They also found high accuracy with $98.87 \%$ specificity for benign conditions and $94.82 \%$ specificity for malignant conditions. In contrast, two studies found vulvar cytology not to be an accurate predictor for histology $[13,19]$. Kashimure and colleagues provided data on vulvar cytology for 55 women with carcinoma of the vulva (Kashimura et al., 1993). They found $11 \%$ had a negative cytology result, $24 \%$ were reported as suspicious, and $65 \%$ were reported as positive; however findings for women with VIN 
were not reported. The low rates for carcinoma of the vulva in this study may be attributed to the keratinization of the tissue, making exfoliation of the malignant cells difficult. The most recent study reported only $32 \%$ of the 22 patients with biopsy proven disease had a positive vulvar cytology test result (Bae-Jump et al., 2007). Both of these studies utilized a scraping technique to obtain their sample. The most recent study was retrospective and therefore sampling errors may have occurred. Overall, vulvar cytology appears to correspond well to histology, however further studies are needed with standardization of collection techniques.

\section{Clinical Pathway Placement}

Research has not been conducted on the role vulvar cytology could play in vulvar disease and screening. Previous studies have focused on technique, correlation with histology, and interpretation of cytology findings. However, clinical expertise can postulate the placement of vulvar cytology in order to evaluate its effectiveness. Screening all women with vulvar cytology does not seem feasible. The incidence of VIN has been reported as $2.1 / 100,000$. This incidence is too small to justify a screening measure and the costs may outweigh the benefit from a health care policy perspective. However, it may be feasible for vulvar cytology to be utilized one of two ways: 1) for those who present with known risk factors for VIN and/or 2) as a screening tool for women with a history of VIN. Risk factors for VIN include: an abnormal cervical cytology, cigarette smoking, immunosuppression, multiple sexual partners, and a history of condyloma, gonorrhea, or herpes simplex (Al-Ghamdi et al., 2002; Brinton et al., 1990; Kurman, Trimble, \& Shah, 1992; Sherman et al., 1991). Women who have a compromised immune system due to disease or steroid use may be at greater risk for VIN and subsequent development of squamous cell carcinoma due to their potential inability to eradicate the HPV infection of the lower genital tract (Al-Ghamdi et al., 2002). In addition, it has been found that approximately half of women with vulvar cancer have lichen sclerosis (Scurry, 1999); therefore, it is imperative that women with lichen sclerosis be screened at regular intervals for the development of vulvar cancer. Vulvar cytology could be an appropriate screening tool for women with these risk factors if an adequate universally accepted technique is found.

It may also be feasible to utilize vulvar cytology to screen women with a history of VIN. As described earlier, recurrence rates have been found to be as high as $52 \%$ (Hillemanns et al., 2006; Jones et al., 2005; Thuis et al., 2000). Vulvar cytology may not only save women from unnecessary vulvar biopsies, but may also be more cost effective. Vulvar cytology is one-fourth the cost of vulvar colposcopy. Studies need to be conducted to determine the cost-benefit ratio for utilizing vulvar cytology in the clinical pathway for evaluation of possible recurrence in these women.

\section{Conclusion}

Cervical cytology is routinely used to screen for cervical cancer and has been extremely successful in reducing the rates of cervical cancer in industrialized countries. 
Years ago, vulvar cytology was used more routinely, however its practice has been largely abandoned today due to issues with air drying, artifact, and inadequate number of cells for evaluation. Former studies showed promise for this screening method and the advent of liquid based cytology may resolve some of the issues related to artifacts and drying. This screening tool deserves to be re-evaluated due for the possible benefits it may provide. It could be a key factor in the early detection of lesions. Vulvar cytology is inexpensive and easily performed. A broader array of health care providers could utilize this screening method and would be more comfortable with this skill set than performing a biopsy. In addition, this may be more cost-effective than initially doing a vulvar biopsy. Further research should be conducted to answer these important questions. 


\section{CHAPTER 3. METHODOLOGY}

As a feasibility study, the findings will help inform future research on sampling technique and accuracy of vulvar cytology. This study will assist in determining if the sampling technique is appropriate and if the knowledge of cytologic interpretation of specimens from the vulva is sensitive and specific to the disease state. These preliminary findings may further enhance clinical care by identifying a non-invasive, less-painful, and potentially more cost effective method for identifying recurrent disease in women with VIN than through tissue biopsy. This could enhance patient compliance for follow up evaluation, therefore potentially detecting disease earlier. The purpose of this study was to determine the feasibility and efficacy of developing a protocol for cytologic sampling of vulvar lesions in conjunction with HPV testing as a valid method to monitor disease status in women being treated and followed for VIN.

\section{Research Design}

The study used a cross-sectional design to determine accuracy of cytology and HPV typing. In addition, correlation was performed among cytology, tissue biopsy, and HPV type in samples taken from women with a history of VIN as compared to a control group of samples. The specific aims and research questions were:

\section{Specific Aim 1 and Research Questions}

To determine the feasibility of a protocol for cytologic sampling of vulvar lesions.

1.1 Does the vulvar sampling technique provide adequate cellularity for cytologic examination?

1.2 What is the sensitivity of vulvar cytology?

1.3 What is the specificity of vulvar cytology?

1.4 What are the negative and positive predictive values of vulvar cytology?

\section{Specific Aim 2 and Research Questions}

To evaluate the efficacy of vulvar cytology in conjunction with Human Papillomavirus (HPV) testing as a valid method to evaluate recurrent or persistent vulvar intraepithelial neoplasia.

2.1 What is the relationship between vulvar cytology results and HPV results?

2.2 Can HPV testing in combination with vulvar cytology, while controlling for age, be utilized as a triage tool for pathology results?

2.3 What is the accuracy of PCR analysis for HPV as a predictor of vulvar intraepithelial neoplasia? 
2.4 If an association is found between cytology/HPV and pathology results, then determine if cytology in conjunction with HPV testing predict the biopsy outcome?

\section{Sample and Setting}

The feasibility study included a total of 48 women, 33 of whom had a history of VIN and presented with a lesion (defined as an acetowhite change following application of acetic acid), and were scheduled for colposcopy with biopsy as part of their planned follow-up management. The remaining 15 women had no prior history of VIN and no visible presentation of lesions and were used as controls. An additional set of "control" specimens were obtained from 15 of the women with a history of VIN from an area that is free from any visible lesions. Women placed in the control group were assumed to be negative for VIN if they do not have a HR HPV on HPV typing. For data analysis, a total of 7 specimen groups were developed under these two groups of women (Figure 2).

Inclusion criteria for the VIN group were:

- female over the age of 18 and under the age of 65

- a history of VIN who present with a visible lesion

- scheduled for colposcopy and possible biopsy as part of their post-treatment management

- ability to speak and read English

Inclusion criteria for the healthy control group were:

- female over the age of 18 and under the age of 65

- scheduled for colposcopy for cervical dysplasia

- absence of any visible vulvar lesions

- ability to speak and read English

Exclusion criteria for the VIN group included patients who present with no lesion at follow-up.

Exclusion criteria for the health control group included:

- a history of VIN

- presentation of visible lesion on examination

Inclusion criteria for the lesion sampled included the sample being deemed adequate by the processing laboratory.

Participants were recruited from an urban community colposcopy clinic. Approximately $80 \%$ of the clientele are minorities (approximately $70 \%$ African 


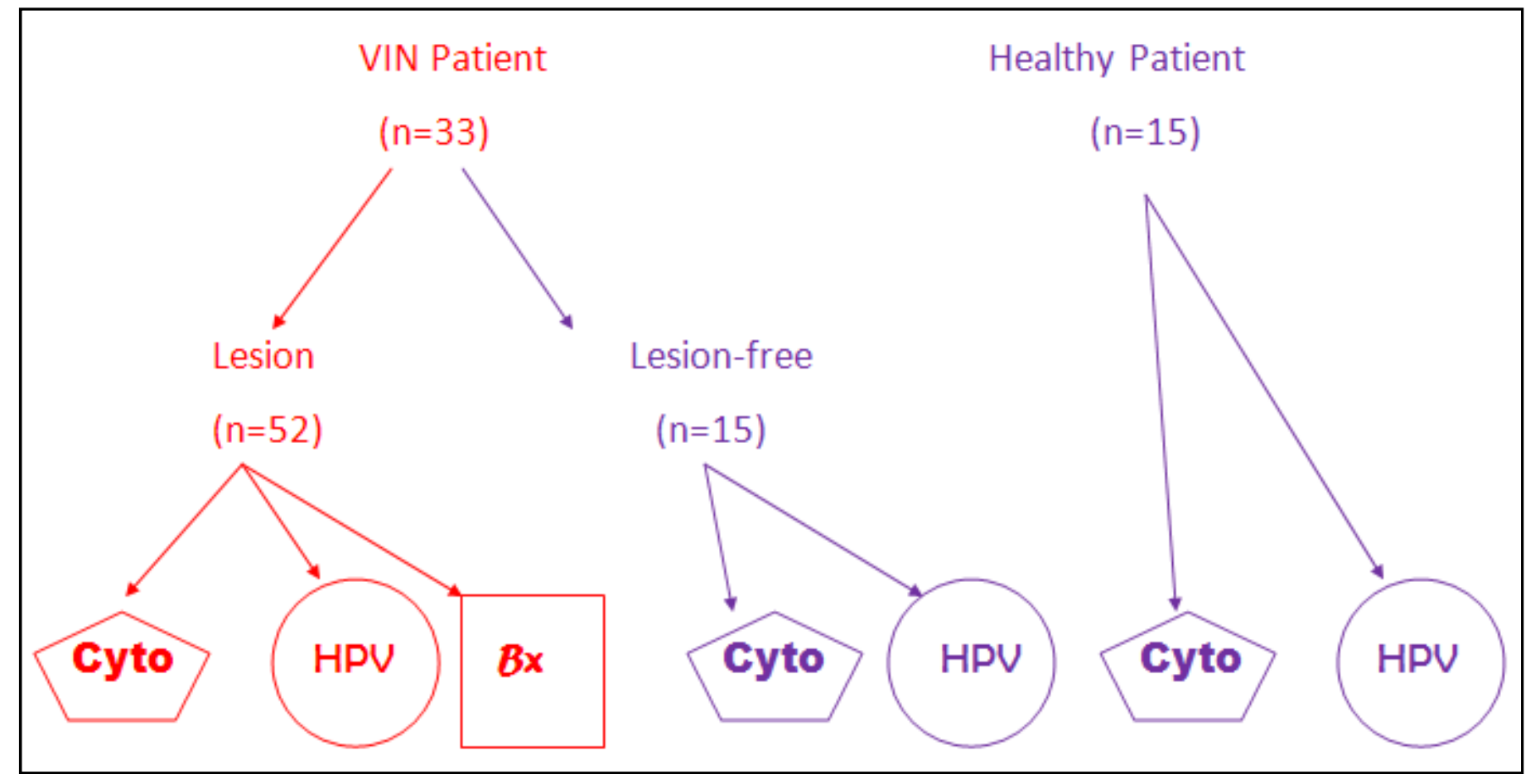

Figure 2. Specimen groups 
American, 5\% Hispanic, and 5\% other) and have Medicaid. This site performs 6-8 vulvar colposcopies per month on women previously diagnosed with VIN. Participants in the VIN study group were recruited upon presentation to the clinic for a scheduled visit after the provider identified that they were eligible and willing to participate, provided a lesion was found that would necessitate biopsy as part of their scheduled management. Healthy patients used as controls were recruited upon presentation to the clinic for a scheduled visit for colposcopy after an abnormal Pap smear. Following an explanation of the study, confidentiality procedures and patient rights were explained. All potential participants were told that they are not obligated to participate and their care would not be affected if they declined to participate. A written informed consent was obtained from the participant if they agreed to participate in the study. Laboratory specimens were sent to two separate facilities. Vulvar cytology and tissue specimens were sent to the local processing laboratory used by the clinical facility. This laboratory is local and is part of a national network of clinical laboratories that perform more than one million tests on approximately 400,000 specimens per day. The HPV samples were sent to an out of state laboratory that provides a full range of quality anatomic, pathology, and consultation services with expertise in HPV typing with the most sensitive HPV primer (PGMY 09/11).

\section{Study Procedures}

All participants in the VIN group received liquid based cytology with HPV testing in addition to their scheduled vulvar biopsy. In 15 of these women, an additional control sample of cytology and HPV testing was taken from an area clinically free from a vulvar abnormality. Participants placed in the healthy control group $(n=15)$ received liquid based cytology and HPV testing only of the vulva. Accuracy was based on sensitivity, specificity and predictive value of the liquid based cytology interpretation and HPV results in relation to the final histologic diagnosis (if tissue biopsy was done) or participant control status. Since tissue biopsy, as a gold standard, was not available from the controls (healthy and VIN controls), they were assumed to be VIN free if they did not have an IR or HR HPV on HPV testing. If they were IR or HR HPV positive, they were excluded as a control since it could not be confirmed if they truly did not have a VIN lesion that was missed during examination.

\section{Specimen Collection}

Colposcopy of the vulva was performed with the application of 5\% acetic acid to the vulva (covering all areas including the rectum) for at least 3-5 minutes. Visual inspection along with a colposcope at $4 \mathrm{x}$ magnification was used to identify any acetowhite lesions. 


\section{Cytology and Human Papillomavirus}

The identified acetowhite lesion was anesthetized with injectable lidocaine and sampled for cytology by scraping cells from the surface of the lesion with a cytobrush collection device that was pre-moistened with sterile saline. This was done two times in order to have samples for cytology and HPV typing. The cytology sample was labeled specimen A and the HPV specimen was labeled B. A different cytobrush and vial was used for each lesion identified as needing a biopsy per clinical judgment. The cytobrush devices were then placed into a vial of PreservCyt ${ }^{\circledR}$ solution for transported to the lab facility. After collection of the cytology sample, a tissue biopsy was performed as part of the regular planned management for the patient. To ensure correlation with cytology, the tissue collected was placed in formalin and transported to the same laboratory facility as the cytology specimen. The procedure described above was also carried out in an area of the vulva that is free from any visible lesions for patients who had a history of VIN and presented with a lesion within another area of the vulva. This procedure was also conducted on healthy controls from the labia majora.

Once received in the laboratory, the PreservCyt ${ }^{\circledR}$ vial for cytology was processed using the ThinPrep ${ }^{\circledR}$ processor, stained with Papanicolaou stain, and cover slipped. A certified cytotechnologist, obtained the specimen from the laboratory facility for evaluation under a light microscope. The specimen was evaluated for adequacy and screened for any abnormalities indicating a malignant or pre-malignant condition. Appropriate cells were identified by marking with a pen and a preliminary interpretation made. The specimen was forwarded to a board certified cytopathologist for final interpretation and reporting of the cytologic diagnosis. The same cytopathologist interpreted all of the specimens for this study.

Specimen vial B was sent to a separate laboratory facility for PCR detection of HPV. Specimens testing positive for HPV detection, were automatically reflexed to PCR identification of high and low risk HPV types. PCR is a selective target amplification assay that generally has a sensitivity of 1-10 copies per PCR reaction. The PGMY09/11 primer was utilized. This primer has the most sensitivity (80-90\%) of the primers for detecting multiple HPV infection (Fuessel et al., 2003; Klug et al., 2008; Nonogaki et al., 2004; Qu et al., 1997). This primer amplifies the conservative L1 region of the HPV genome which generates a single DNA strand. This strand is of a particular prescribed molecular weight specific for each HPV type present. Restricted fragment length polymorphism (RFLP) analysis of the PCR products creates unique band patterns utilized in HPV type identification.

\section{Tissue Biopsy}

After collection of the cytology sample from the acetowhite lesion, a tissue biopsy was performed as part of the regular planned management for the patient. Tissue biopsy was conducted by cleaning the area with betadine (if not allergic) and anesthetizing with injected lidocaine. A 3-5mm keys punch biopsy was used to obtain the tissue specimen. 
Homeostasis was achieved using silver nitrate. The patient was instructed not to rub the area and to keep clean and dry for one week. To ensure correlation with cytology, the tissue collected was placed in formalin and transported to the same laboratory facility as the cytology specimen. Upon receipt of the biopsy specimen(s) in the laboratory facility, the specimen was processed, stained with Hematoxylin and Eosin, and cover slipped. The cytopathologist obtained the specimen for evaluation and diagnosis. The cytopathologist was blinded to the VIN status of participants. The histology report was sent to the patient's provider to be included in the patient's medical records.

\section{Data Analysis}

Demographic data of race and age was collected to describe the sample. Results of cytologic interpretation, HPV testing and biopsy diagnosis were recorded in an Excel database and imported into SAS for correlation and analysis using descriptive statistics. Since some participants selected as controls had an abnormal cytology or positive HPV test, further identification of controls occurred. A participant was removed as a control if they had a HR HPV identified and were not included in the data analysis for sensitivity, specificity, and predictive value of vulvar cytology and HPV testing. This resulted in 6 participants being removed as controls from this portion of the analysis.

To answer Aim 1.1, a proportion was used to determine if an adequate number of cytologic specimens had appropriate cellularity for examination. An adequate number of cells was defined as 3-4 cells per 40X high power field. The cytology must have been deemed adequate to have a cytologic interpretation appropriate for analysis. Nine specimens were deemed inadequate for evaluation and removed from further analysis.

To answer Aim 1.2-1.4, cytology and HPV testing from VIN and healthy women were categorized as either positive or negative (Figure 2). Biopsy specimens were likewise categorized. For purposes of analysis, the gold standard for which cytology was compared was established by HPV- status (being negative for IR or HR HPV) or biopsy. The specimen was categorized as positive for the disease state if the biopsy was positive for VIN 1, 2, or 3. The cytology specimen group was formed by including all cytology specimens from the VIN lesions, VIN-free lesions (in women with a history of VIN), and healthy controls. Cytology results of ASCUS or greater were considered positive and negative results remained negative. This resulted in four groups. These 4 groups (VIN +, VIN -, cytology +, or cytology -) were placed into a $2 \times 2$ table for analysis. Six specimens were removed due to a HR HPV in a control sample and an additional 9 specimens were removed due to lack of cellularity, leaving 67 total specimens for evaluation. Evaluation of the adequacy of vulvar cytology for VIN was then calculated.

Sensitivity refers to the proportion of positive test results that are truly positive. Specificity is the proportion of negatives that are truly negative. Positive predictive value was determined by evaluating the proportion of true positives among those reported positive. Negative predictive value was calculated by the proportion of true negatives Among those reported negative. 
To answer Aim 2.1, HPV results were converted to categorical data of negative (0), low risk (1), intermediate risk/ high risk (2), and unknown (3). A nonparametric test was used for the analysis since the sample was small and data were categorical. Fisher's exact was used to determine the association of HPV results and cytology results.

To answer Aim 2.2 a logistic regression was used with the biopsy outcome serving as the dependent variable (therefore only those with a tissue biopsy was included in the analysis) and the HPV/cytology results as the independent variable while controlling for age. Age was controlled for since higher mean ages have been associated with vulvar cancer and well-differentiated VIN which tends to be more aggressive (Haefner, Tate, McLachlin, \& Crum, 1995).

To answer Aim 2.3, sensitivity, specificity, positive predictive value, and negative predictive value was calculated on HPV analysis as a predictor of VIN. HPV testing from VIN and healthy women were categorized as either positive or negative (Figure 2).

Biopsy specimens were likewise categorized. For purposes of analysis, the gold standard for which cytology was compared was established by HPV- status (being negative for IR or HR HPV) or biopsy. The specimen was categorized as positive for the disease state if the biopsy was positive for VIN 1, 2, or 3.The HPV specimen group was formed by including all HPV specimens from the VIN lesions, VIN-free lesions (in women with a history of VIN), and healthy controls. HPV results of IR or HR HPV were considered positive. LR and negative results remained negative. This resulted in four groups. These 4 groups (VIN +, VIN -, HPV +, or HPV -) were placed into a $2 \times 2$ table for analysis. Six specimens were removed due to a HR HPV in a control sample, 1 specimen was removed because of an unknown HPV type, and an additional 16 specimens were removed due to lack of cellularity, leaving 59 total specimen samples for evaluation. Evaluation of the adequacy of vulvar cytology for VIN was then calculated.

Aim 2.4 would have been answered using a ROC curve to determine the ability of cytology in conjunction with HPV testing to predict the outcome of VIN. Aim 2.4 would have only been conducted if an association was found between cytology/HPV and pathology results as completed for Aim 2.2. No power analysis was performed as this is a feasibility study of a rare disease. The planned number of participants is consistent with the number of subjects in other pilot studies in this area (Levine et al., 2001).

\section{Protection of Human Subjects}

This study was approved by the University of Tennessee Health Science Center Institutional Review Board (see Appendix A). Potential study participants were identified by their healthcare provider to determine if they met the inclusion and exclusion criteria. If they met inclusion criteria, the study was explained in detail by a member of the research team and participants were provided with a copy of the consent form (see Appendix B). Potential participants were told that they were not obligated to participate and their care would not be affected if they declined to participate. After individuals reviewed the consent form at their leisure, the contents of the consent form were re- 
reviewed and any questions answered. Written consent was obtained and a copy of the signed consent form was given to the participant.

The risks associated with the cytologic sampling required for this study procedure included possible irritation from the cytobrush. The vulvar area was anesthetized prior to cytologic sampling to reduce the amount of discomfort experienced during the procedure. Risks associated with vulvar biopsy (the customary and usual practice for diagnosing vulvar lesions) include pain, bleeding, and infection. The participant was advised of the possibility of discomfort prior to consenting to the study and was advised to notify their healthcare providers if they experienced any vulvar problems.

The research record and specimens were labeled with a code number. A master key that linked the subject's name to the code number was maintained in a separate and secure location. The patient's name and identifying information was used in accordance with standard policy for receipt of specimens in the laboratory. HIPAA authorization was included in the confidentiality section of the consent form. No individual identifying information will be used when conclusions and results are reported or presented for scientific review. 


\section{CHAPTER 4. RESULTS AND CONCLUSIONS}

\section{Introduction}

Vuvlar intraepithelial neoplasia (VIN), a rare skin condition that may lead to vulvar cancer, is increasing in incidence. Studies have found VIN has doubled in all age groups, but tripled in women under the age of 50 (Joura, Haider-Angeler, Breitenecker, \& Leodolter, 2000; Sturgeon et al., 1992). In addition, vulvar cancer has increased by $20 \%$ in the United States between 1973 and 2000 (Judson et al., 2006). These increases are attributed to increasing rates of human papillomavirus (HPV), a sexually transmitted virus. The recurrence rate for VIN has been found to be as high as 35\%. Currently the gold standard for evaluation of VIN recurrence is vulvar colposcopy and tissue biopsy. Although tissue biopsy is the gold standard, vulvar colposcopy is difficult to perform on the vulva. Acetowhite changes are non-specific and many health care providers are not well versed in performing this procedure. Various skin conditions may mimic VIN and therefore all lesions are typically subjected to tissue evaluation through biopsy. As a result, many women are subjected to tissue biopsies that may not be warranted. Tissue biopsy is painful and anxiety producing. Women with VIN have specifically reported that stress and anxiety accompany their clinical exams and anticipation of a vulvar biopsy (Likes et al., 2008).

Currently tissue biopsy is the only tool utilized in diagnosing VIN. The use of a triage test may decrease un-necessary painful tissue biopsies in these women who will undergo continuous evaluation with the possibility of many biopsies over their lifetime. Cytology (Pap smear) has been highly successful in the triage of patients with cervical cancer and cervical dysplasia, however in the past it has failed to show similar success rates on the vulva. This difficulty results from air drying, artifact, keratinization of the skin making interpretation difficult, and inadequate collection techniques to obtain sufficient numbers of cells for evaluation. However, with the development of liquidbased cytologic technology, the use of vulvar cytology merits re-evaluation of this technique. Vulvar cytology offers several advantages of tissue biopsy in that it is less invasive, less painful, and may be more cost effective. In addition, vulvar cytology has the ability to sample a larger area of the vulva than the $3-4 \mathrm{~mm}$ area taken by a punch biopsy. Therefore, the purpose of this pilot study was to determine the feasibility of cytologic sampling of vulvar lesions and to evaluate the efficacy of vulvar cytology in conjunction with HPV testing as a valid method for following women treated for VIN. Specific study aims and research questions were:

\section{Specific Aim 1 and Research Questions}

To determine the feasibility of a protocol for cytologic sampling of vulvar lesions.

1.1 Does the vulvar sampling technique provide adequate cellularity for cytologic examination?

1.2 What is the sensitivity of vulvar cytology? 
1.3 What is the specificity of vulvar cytology?

1.4 What are the negative and positive predictive values of vulvar cytology?

\section{Specific Aim 2 and Research Questions}

To evaluate the efficacy of vulvar cytology in conjunction with Human Papillomavirus (HPV) testing as a valid method to evaluate recurrent or persistent vulvar intraepithelial neoplasia.

2.1 What is the relationship between vulvar cytology results and HPV results?

2.2 Can HPV testing in combination with vulvar cytology, while controlling for age, be utilized as a triage tool for tissue biopsy results?

2.3 What is the accuracy of PCR analysis for HPV as a predictor of vulvar intraepithelial neoplasia?

2.4 If an association is found between cytology/HPV and tissue biopsy results, then determine if cytology in conjunction with HPV testing predict the biopsy outcome?

\section{Materials and Methods}

A cross-sectional design was used to determine the correlation among cytology, tissue biopsy, and HPV type in women with a history of VIN and a clinically suspicious lesion as compared to a control group. Two sets of women were included in the control group. One set consisted of clinically apparent VIN- free women and the second set included samples from of an area clinically free of possible VIN in women with a history of VIN. The samples collected as control specimens were assumed to be negative for VIN if the HPV testing results were negative for HR HPV. If they were positive for HR $\mathrm{HPV}$, they were excluded from analysis of sensitivity, specificity, and predictive value $(\mathrm{n}=6)$. Forty-eight women were recruited from a community colposcopy clinic and provided informed consent for this Institutional Review Board study, with a total of 82 specimen collected for cytology/HPV. The patient population from this community colposcopy clinic includes a high percentage (approximately $35 \%$ ) of immunocompromised patients, therefore at a higher risk for VIN and recurrence of VIN. Although a power analysis was not performed for this feasibility study, the number of participants is consistent with the number of participants in other pilot studies in this area (Levine, 2001).

After signed informed consent, vulvar colposcopy with 5\% acetic acid and visual inspection was performed to assess the absence or presence of a clinically suspicious vulvar lesion. Once a clinically suspicious lesion was identified, it was anesthetized with injectable lidocaine and sampled for cytology by scraping the cells from the surface of the lesion with a saline moistened cytobrush collection device rotated 20 times. The procedure was repeated with a second cytobrush for HPV typing through PCR. The cytobrushes were placed in separate vials of PreservCyt $\mathbb{R}$ solution for transport to the 
appropriate laboratory facility. Tissue biopsy of the lesion was then obtained using a 3$5 \mathrm{~mm}$ keys punch biopsy. In the control specimens, the same procedures were used, however the injection of lidocaine was not performed since no tissue biopsy was obtained. The pathologist and cytotechnologist were blinded to the VIN status of the participant. A total of 82 specimen groups were collected from these 48 women. Fiftytwo of these specimens were obtained from VIN-suspicious lesions in women with a history of VIN. An additional 15 specimens, to be used as controls, were taken from these women with VIN in an area of the vulva with no gross abnormalities. Additional control specimens were taken from 15 women with no history of VIN and no gross vulvar abnormalities.

The PreservCyt ${ }^{\circledR}$ vial for cytology was processed using the ThinPrep ${ }^{\circledR}$ processor, stained with Papanicolaou stain, and cover slipped. The specimen was screened for cellular adequacy and screened for possible malignant or premalignant abnormalities. A certified cytotechnologist and board certified cytopathologist provided a final cytologic interpretation. The second PreservCyt ${ }^{\circledR}$ vial was sent for PCR detection for HPV. If PCR testing was positive for HPV, the specimen was HPV typed using PGMY 09/11 primer. PGMY 09/11 primer has the most sensitivity (80-90\%) of the primers and identifies 50 HPV types through amplification of the L1 region of the HPV genome (Figure 3).

\section{Statistical Analysis}

Results of cytologic interpretation, HPV testing, and biopsy diagnosis were recorded in an Excel database and imported into SAS for analysis using descriptive statistics. Since some specimens selected as controls had abnormal cytology or positive HPV tests, further identification of controls continued to occur until an adequate number of controls was obtained. A specimen was classified as a control and removed from further analysis of sensitivity, specificity, and predictive value if they had a HR HPV identified $(n=6)$. These controls were assumed negative for VIN since tissue biopsy was not available. Sensitivity and specificity of cytology and HPV typing were compared to tissue biopsy and/or control (automatically assumed negative for disease) and placed in a $2 \times 2$ table for analysis. Positive predictive values were determined by evaluating the proportion of true positives among those reported positive. Negative predictive values were determined by evaluating the proportion of true negatives among those reported negative. HPV results were converted into categorical values of negative, low risk, intermediate risk/high risk, or unknown and a Fisher's Exact test used to determine the association of HPV and cytology results. If multiple HPV types were present, the specimen was coded with the highest risk HPV. For example, if a lesion had two LR HPV types and a HR HPV type, they were coded as HR HPV. A logistic regression with the biopsy result as the dependent variable and cytology/HPV results as the independent variables were used to determine if cytology in combination with HPV could be used as a triage tool for pathology results. Age was controlled for in this regression analysis since it has been found that with increasing age, VIN may be more aggressive. If an association would have been found between cytology/HPV results and pathology results, a ROC curve would have been performed. 


\begin{tabular}{|lllll|}
\hline 6 & 34 & 52 & 62 & 75 \\
11 & 35 & 53 & 64 & 77 \\
16 & 39 & 54 & 66 & 80 \\
18 & 40 & 55 & 67 & 81 \\
26 & 42 & 56 & 68 & 82 \\
30 & 43 & 57 & 69 & 83 \\
31 & 44 & 58 & 70 & 84 \\
32 & 45 & 59 & 72 & CP6108 \\
33 & 49 & 60 & 73 & CP8061 \\
33 & 51 & 61 & 74 & LVX160 \\
\hline
\end{tabular}

Figure 3: HPV types by PGMY 09/11 premier 


\section{Results}

The study consisted of 82 samples from 48 women with a mean age of 34 (range 19-65). Demographics of each group can be found in Table 1. Of the 52 tissue samples, $32(62 \%)$ were reported as VIN I. ASCUS and VIN I were each reported in 33\% $(n=27)$ of cytology samples. The cytology, HPV, and when appropriate, histology results from the 82 lesions are reported in Table 2.

Cytology results are reported as quantity not sufficient (QNS), negative, ASCUS, ASCUS favor VIN 1,VIN 1, VIN 1/VIN 2, VIN 2, or VIN 2/VIN 3. The HPV results are reported as negative, low risk, intermediate risk/ high risk, or unknown. When coding for HPV status, the HPV result with the highest risk was deferred to for each lesion. For example, if a lesion had two LR HPV types and one HR HPV type identified, the lesion was coded as positive for HR HPV. Pathology, when available, is reported as negative (defined as non-dysplastic and includes hyperplasia), VIN I (including condyloma), VIN II, or VIN III. Since some specimens selected as controls had positive HR HPV tests, further identification of controls was conducted. A specimen was classified as a control if they had a negative biopsy or were HR HPV negative. The control specimens that were positive for a HR HPV were not used in the data analysis for sensitivity, specificity, or predictive value. This resulted in 6 specimens being excluded from these analyses.

\section{Aim 1.1: Adequacy}

An adequate number of cells for cytologic interpretation were defined as 3-4 cells per 40X high power field. An adequate number of cells for cytologic samples were found in $90 \%$ (74 of 82) of samples. Adequacy was less frequent for HPV PCR with $72 \%$ (59 of 82) samples being adequate for evaluation.

\section{Aims 1.2-1.4 and Aim 2.3: Sensitivity, Specificity and Predictive Value}

Of the 82 specimens, 68 were available for evaluation of vulvar cytology specificity, sensitivity, and predictive value, 8 were inadequate for evaluation due to lack of cellularity, and 6 were removed from analysis due to the control returning positive for a HR HPV. Vulvar cytology achieved a sensitivity of $95 \%$ with a trade-off of only $15 \%$ specificity. Positive and negative predictive value was 65\% (40 true positives and 22 false positives) and $67 \%$ respectively (4 true negatives and 2 false negative). HPV PCR achieved $62 \%$ sensitivity and $85 \%$ specificity. Positive and negative predictive value was $89 \%$ ( 24 true positives and 3 false positives) and 53\% (17 true negative and 15 false negatives) respectively. The $2 \times 2$ tables used for sensitivity and specificity can be found in Tables 3 and 4. 
Table 1. Demographics

\begin{tabular}{lccc}
\hline Demographics & All Women & VIN & Controls \\
\hline Age & $34(19-65)$ & $35(19-65)$ & $33(19-62)$ \\
Mean (range) & & & \\
Ethnicity (n) & 43 & 31 & 25 \\
$\quad \begin{array}{l}\text { African } \\
\text { American }\end{array}$ & 4 & 1 & 5 \\
Caucasian & 1 & 1 & 0 \\
Other & & & \\
\hline
\end{tabular}


Table 2. Participant lesion results

\begin{tabular}{|c|c|c|c|c|}
\hline $\begin{array}{c}\text { Participant } \\
\#\end{array}$ & $\begin{array}{l}\text { Lesion } \\
\#\end{array}$ & Cytology & HPV & Tissue Biopsy \\
\hline 1 & 1 & QNS* & Negative & Squamous hyperplasia \\
\hline 1 & 2 & QNS & QNS & VIN 1 \\
\hline 2 & 3 & VIN 1 & $\mathrm{HR}^{*}$ & VIN 1 \\
\hline 2 & 4 & Negative & HR & VIN 1 \\
\hline 2 & 5 & ASCUS-favor VIN 1 & HR & VIN 1 \\
\hline 2 & 6 & ASCUS & QNS & NA \\
\hline 2 & 7 & ASCUS & Unknown & VIN 1 \\
\hline 3 & 8 & ASCUS- favor VIN 1 & Negative & VIN 1 \\
\hline 4 & 9 & VIN 1/VIN 2 & $\mathrm{HR}$ & VIN 2 \\
\hline 4 & 10 & VIN 1 & HR & VIN 1 \\
\hline 5 & 11 & VIN 1/VIN 2 & HR & VIN 3 \\
\hline 5 & 12 & QNS & HR & VIN 2 \\
\hline 5 & 13 & VIN 1/VIN 2 & LR & VIN 2 \\
\hline 6 & 14 & Negative & HR & VIN 1 \\
\hline 6 & 15 & VIN 1 & HR & Negative \\
\hline 7 & 16 & VIN 1 & Negative & VIN 1 \\
\hline 8 & 17 & VIN 1 & HR & VIN 1 \\
\hline 8 & 18 & VIN 1 & $\mathrm{HR}$ & VIN 1 \\
\hline 9 & 19 & VIN 1 & Negative & VIN 1 \\
\hline 10 & 20 & VIN 1 & Negative & VIN 1 \\
\hline 11 & 21 & VIN 1 & $\mathrm{LR}^{*}$ & VIN 1 \\
\hline 11 & 22 & VIN 1 & LR & VIN 1 \\
\hline 12 & 23 & VIN 1 & LR & VIN 1 \\
\hline 12 & 24 & VIN 1 & LR & VIN 1 \\
\hline 12 & 25 & ASCUS & LR & Hyperplasia \\
\hline 12 & 26 & ASCUS & LR & NA* \\
\hline 13 & 27 & VIN 1 & HR & VIN 1 \\
\hline 14 & 28 & ASCUS & Negative & VIN 1 \\
\hline 15 & 29 & VIN 1/ VIN 2 & Negative & VIN 1 \\
\hline 16 & 30 & VIN 1 & LR & VIN 1 \\
\hline 16 & 31 & ASCUS & QNS & VIN 1 \\
\hline 16 & 32 & ASCUS & Negative & NA \\
\hline 17 & 33 & VIN 1 & HR & VIN 1 \\
\hline 18 & 34 & VIN 1/ VIN 2 & LR & VIN 1 \\
\hline 19 & 35 & ASCUS & QNS & Negative \\
\hline
\end{tabular}


Table 2. (continued)

\begin{tabular}{|c|c|c|c|c|}
\hline $\begin{array}{c}\text { Participant } \\
\#\end{array}$ & $\begin{array}{l}\text { Lesion } \\
\quad \#\end{array}$ & Cytology & HPV & Tissue Biopsy \\
\hline 19 & 37 & QNS & QNS & VIN 1 \\
\hline 19 & 38 & QNS & QNS & NA \\
\hline 20 & 39 & ASCUS favor VIN 1 & HR & Hyperplasia \\
\hline 20 & 40 & ASCUS & HR & NA \\
\hline 20 & 41 & QNS & Negative & VIN 1 \\
\hline 21 & 42 & VIN 1/ VIN 2 & $\mathrm{HR}$ & VIN 2 \\
\hline 21 & 43 & VIN 1 & HR & VIN 1 \\
\hline 21 & 44 & ASCUS & QNS & NA \\
\hline 22 & 45 & VIN 1 & Negative & NA \\
\hline 23 & 46 & Negative & Negative & NA \\
\hline 24 & 47 & VIN 1/ VIN 2 & HR & VIN 1 \\
\hline 24 & 48 & VIN 1 & LR & NA \\
\hline 25 & 49 & VIN 1 & HR & VIN 1 \\
\hline 25 & 50 & VIN 1 & HR & NA \\
\hline 26 & 51 & ASCUS & HR & VIN 2 \\
\hline 26 & 52 & VIN 1 & HR & VIN 1 \\
\hline 27 & 53 & Negative & QNS & NA \\
\hline 28 & 54 & ASCUS & LR & NA \\
\hline 29 & 55 & ASCUS & LR & NA \\
\hline 30 & 56 & ASCUS & Negative & NA \\
\hline 31 & 57 & ASCUS & QNS & VIN 2 \\
\hline 32 & 58 & VIN 1/ VIN 2 & Negative & Negative \\
\hline 33 & 59 & VIN 1 & $\mathrm{HR}$ & VIN 2 \\
\hline 33 & 60 & ASCUS & LR & VIN 2 \\
\hline 34 & 61 & ASCUS & Negative & Hyperplasia \\
\hline 34 & 62 & ASCUS & QNS & NA \\
\hline 35 & 63 & VIN 1 & $\mathrm{IR}^{*}$ & VIN 1 \\
\hline 35 & 64 & QNS & QNS & NA \\
\hline 36 & 65 & Negative & LR & NA \\
\hline 37 & 66 & VIN 1 & LR & NA \\
\hline 38 & 67 & VIN 1/ VIN 2 & HR & NA \\
\hline 38 & 68 & VIN 2/ VIN 3 & HR & VIN 3 \\
\hline 39 & 69 & ASCUS & Negative & NA \\
\hline 40 & 70 & Negative & Negative & NA \\
\hline 41 & 71 & ASCUS & HR & NA \\
\hline
\end{tabular}


Table 2. (continued)

\begin{tabular}{lllll}
\hline $\begin{array}{c}\text { Participant } \\
\#\end{array}$ & $\begin{array}{c}\text { Lesion } \\
\#\end{array}$ & Cytology & HPV & Tissue Biopsy \\
\hline 42 & 72 & VIN 1 & HR & NA \\
43 & 73 & ASCUS & HR & VIN 2/ VIN 3 \\
43 & 74 & ASCUS & HR & NA \\
44 & 75 & VIN 1 & Negative & NA \\
45 & 76 & VIN 2/ VIN 3 & HR & VIN 3 \\
45 & 77 & VIN 1/ VIN 2 & HR & NA \\
45 & 78 & VIN 1/ VIN 2 & HR & VIN 3 \\
46 & 79 & VIN 1/ VIN 2 & QNS & VIN 1 \\
46 & 80 & QNS & QNS & NA \\
47 & 81 & ASCUS & QNS & NA \\
48 & 82 & VIN 1 & QNS & NA \\
\hline \multicolumn{2}{l}{ Notes: *QNS } \\
intermediate risk; LR = low risk HPV; NA= was a control and no biopsy collected
\end{tabular}


Table 3. Comparison of cytology test results to biopsy results

\begin{tabular}{lccc}
\hline \multirow{2}{*}{ Cytology } & \multicolumn{2}{c}{ Biopsy } & Total \\
\cline { 2 - 3 } & Positive & Negative & \\
\hline Positive & $40(\mathrm{TP})$ & $22(\mathrm{FP})$ & 62 \\
Negative & $2(\mathrm{FN})$ & $4(\mathrm{TN})$ & 6 \\
Total & 42 & 26 & 68 \\
\hline
\end{tabular}


Table 4. Comparison of HPV test results to biopsy results

\begin{tabular}{lccc}
\hline HPV & \multicolumn{2}{c}{ Biopsy } & Total \\
\cline { 2 - 3 } & Positive & Negative & \\
\hline Positive & $24(\mathrm{TP})$ & $3(\mathrm{FP})$ & 27 \\
Negative & $15(\mathrm{FN})$ & $17(\mathrm{TN})$ & 32 \\
Total & 39 & 20 & 59 \\
\hline
\end{tabular}




\section{Aim 2.1 and 2.2: Association of Cytology to HPV PCR / Pathology}

After categorizing cytology results into 4 categories (negative, ASCUS, LGSIL, or HGSIL) and HPV PCR into 4 categories (negative, low risk, intermediate risk/ high risk, and unknown) no association was found between these variables $(p=0.3559)$. A logistic regression analysis was performed, controlling for age, with pathology result as the dependent variable and cytology and HPV PCR as the independent variables. Utilizing forward selection, cytology and HPV PCR were not found to be predictors for pathologic diagnosis of VIN ( $>$ >.05) and fell from the logistic model.

\section{Discussion and Conclusions}

Both the incidence of VIN and vulvar cancer has been increasing. As more women are experiencing the anxieties and risks associated with VIN surveillance and possible recurrence, it is critical to evaluate novel techniques that may decrease their anxiety and facilitate the early identification of recurrent or persistent disease. The collection technique utilized in this study provided for adequate cellularity in the majority of the swabs ( $90 \%$ for cytology and $72 \%$ for HPV PCR). The cytology adequacy rate from this method of collection is consistent with the literature (Levine et al., 2001; Maclean, 2006), however the adequacy rate for HPV was slightly lower than that for the cytology specimens. This difference may have been influenced by the fact that brush sample for the HPV PCR lab was collected following collection of the sample for cytology lab. The cellular material may be more difficult to obtain with the second swab, thus decreasing the adequacy rate in the HPV PCR samples. This study found that by vigorous brushing with a cyto brush pre-moistened with saline, it is possible to obtain an adequate cellular sample from the vulva for cytologic and/or molecular evaluation for HPV. Previous problems with air drying or harvesting cells were not an issue in this study.

The sensitivity of vulvar cytology was high at $95 \%$, however the specificity was low $(15 \%)$. Vulvar cytology also had a low positive predictive value at $65 \%$. Although, vulvar cytology identified the majority of abnormalities, the specificity of the test would yield a substantial number of false positives. This means that the use of cytology would not spare women the need for biopsy. The specificity may be improved through development of standardized criteria for classifying vulvar cytologic specimens. However, this has yet to be shown and should be investigated further. Sensitivity and specificity was moderate to high in range for HPV PCR (62\% and $85 \%$ respectively). Neither cytology nor HPV were found to be predictors for the diagnosis of VIN. This may be due to the specificity issues with vulvar cytology. Again, this may be different with more stringent and clearer criteria for classifying vulvar cytology specimens.

It was of additional interest to evaluate the utility of combining cytology and HPV. If a cytology returned ASCUS, the HPV results were utilized as a reflux test to determine if cytology would be deemed positive or negative. A HPV result of HR HPV resulted in a interpretation of positive cytology, thus ultimately leading to the impression of a needed biopsy. A HPV result of negative or LR HPV was interpreted as a negative 
cytology finding. Fifty-six samples were available for analysis. The sensitivity was $90 \%$, specificity $61 \%$, PPV 83\%, and NPV 73\% (Table 5). The specificity improved, however this was at the expense of sensitivity.

The results of this study may be underestimated due to the selection of controls and the absence of a tissue biopsy in these women to confirm the absence of HPV. Eight of the 27 controls had a LR HPV that did not exclude them as a control. Field effect from their HPV infection may have caused an HPV effect on the vulva, leading to an ASCUS or VIN cytology finding. Without tissue biopsy, this factor and its effect on the specificity of this study could not be verified. However, cytology did not miss any of the VIN 2 or VIN 3 samples. From a clinical perspective, this is critical because we do not treat VIN 1 since it is an overcalled and non-reproducible finding and thought to be a common condition that does not lead to cancer in the majority of women. All the women in the study with VIN 2 or VIN 3 would have been discovered through cytology and treatment initiated. The major limitation of vulvar cytology at this time is specificity and the high false positive rate if, in fact, no "filed effect" is present. This would lead to unnecessary biopsies in these women and decrease the clinical utility of vulvar cytology for surveillance of women with VIN.

In conclusion, because of the anxiety and discomfort related to vulvar biopsy, and the frequency with which biopsies are required to monitor women with a history of VIN, continued evaluation of vulvar cytology in the context of liquid based cytology is warranted. Although this study found positive results with regard to sensitivity, the specificity was poor and did not demonstrate a role for vulvar cytology at this time. However, our study size was limited by the small size. A larger study, after identifying a clearer classification profile for vulvar cytology and confirming the absence of disease, would be important to better address the needs of this growing patient population. 
Table 5. Comparison of cytology/HPV reflux test results to biopsy results

\begin{tabular}{lccc}
\hline Cytology/ HPV & \multicolumn{2}{c}{ Biopsy } & Total \\
\cline { 2 - 3 } & Positive & Negative & \\
\cline { 2 - 3 } Positive & $34(\mathrm{TP})$ & $7(\mathrm{FP})$ & 41 \\
Negative & $4(\mathrm{FN})$ & $11(\mathrm{TN})$ & 15 \\
Total & 38 & 18 & 56 \\
\hline
\end{tabular}

Notes: Sensitivity $=90 \%$; Specificity $=61 \% ; \mathrm{PPV}=83 \%$; NPV= 73\% 


\section{LIST OF REFERENCES}

Al-Ghamdi, A., Freedman, D., Miller, D., Poh, C., Rosin, M., Zhang, L., et al. (2002). Vulvar squamous cell carcinoma in young women: a clinicopathologic study of 21 cases. Gynecologic oncology, 84(1), 94-101.

Ayala, M. J., Vilaplana, E., Pereira, A., Perez-Barroso, M., Granados, M., Pardo, F. S., et al. (1976). Cytodiagnosis of lesions of the vulva (author's transl). Archives d'Anatomie et de Cytologie Pathologiques, 24(5), 339-345.

Bae-Jump, V. L., Bauer, M., \& Van Le, L. (2007). Cytological evaluation correlates poorly with histological diagnosis of vulvar neoplasias. Journal of Lower Genital Tract Disease, 11(1), 8-11.

Brinton, L. A., Nasca, P. C., Mallin, K., Baptiste, M. S., Wilbanks, G. D., \& Richart, R. M. (1990). Case-control study of cancer of the vulva. Obstetrics and Gynecology, 75(5), 859-866.

Carter, B., Kaufmann, L. A., Cuyler, W. K. (1950). Smear preparation in the diagnosis of vulvar carcinoma. Surgery, Gynecology \& Obstetrics, 91, 600-604.

Dennerstein, G. J. (1968). The cytology of the vulva. The Journal of Obstetrics and Gynaecology of the British Commonwealth, 75(6), 603-609.

Dennerstein, G. J. (1988). Cytology of the vulva. Journal of Reproductive Medicine, 33(8), 703-704.

Doorbar, J. (2006). Molecular biology of human papillomavirus infection and cervical cancer. Clinical Science (London, England), 110(5), 525-541.

Fuessel, S., Sickert, D., Meye, A., Klenk, U., Schmidt, U., Schmitz, M., et al. (2003). Multiple tumor marker analyses (PSA, hK2, PSCA, trp-p8) in primary prostate cancers using quantitative RT-PCR. International Journal of Oncology, 23(1), 221-228.

Haefner, H. K., Tate, J. E., McLachlin, C. M., \& Crum, C. P. (1995). Vulvar intraepithelial neoplasia: age, morphological phenotype, papillomavirus DNA, and coexisting invasive carcinoma. Human Pathology, 26(2), 147-154.

Hillemanns, P., Wang, X., Staehle, S., Michels, W., \& Dannecker, C. (2006). Evaluation of different treatment modalities for vulvar intraepithelial neoplasia (VIN): $\mathrm{CO}(2)$ laser vaporization, photodynamic therapy, excision and vulvectomy. Gynecologic Oncology, 100(2), 271-275.

Insinga, R. P., Dasbach, E. J., Elbasha, E. H., Puig, A., \& Reynales-Shigematsu, L. M. (2007). Cost-effectiveness of quadrivalent human papillomavirus (HPV) 
vaccination in Mexico: a transmission dynamic model-based evaluation. Vaccine, 26(1), 128-139.

Jimenez-Ayala, M., \& Jimenez-Ayala, B. (2002). Terminology for vulvar cytology based on the Bethesda System. Acta Cytologica, 46(4), 645-650.

Jones, R. W., Baranyai, J., \& Stables, S. (1997). Trends in squamous cell carcinoma of the vulva: the influence of vulvar intraepithelial neoplasia. Obstetrics and Gynecology, 90(3), 448-452.

Jones, R. W., Rowan, D. M., \& Stewart, A. W. (2005). Vulvar intraepithelial neoplasia: aspects of the natural history and outcome in 405 women. Obstetrics and Gynecology, 106(6), 1319-1326.

Joura, E. A., Losch, A., Haider-Angeler, M. G., Breitenecker, G., \& Leodolter, S. (2000). Trends in vulvar neoplasia. Increasing incidence of vulvar intraepithelial neoplasia and squamous cell carcinoma of the vulva in young women. Journal of Reproductive Medicine, 45(8), 613-615.

Judson, P. L., Habermann, E. B., Baxter, N. N., Durham, S. B., \& Virnig, B. A. (2006). Trends in the incidence of invasive and in situ vulvar carcinoma. Obstetrics and Gynecology, 107(5), 1018-1022.

Kagie, M. J., Kenter, G. G., Zomerdijk-Nooijen, Y., Hermans, J., Schuuring, E., Timmers, P. J., et al. (1997). Human papillomavirus infection in squamous cell carcinoma of the vulva, in various synchronous epithelial changes and in normal vulvar skin. Gynecologic Oncology, 67(2), 178-183.

Kanodia, S., Fahey, L. M., \& Kast, W. M. (2007). Mechanisms used by human papillomaviruses to escape the host immune response. Current Cancer Drug Targets, 7(1), 79-89.

Kashimura, M., Matsuura, Y., Kawagoe, T., Toki, N., \& Sugihara, K. (1993). Cytology of vulvar squamous neoplasia. Acta Cytologica, 37(6), 871-875.

Klug, S. J., Molijn, A., Schopp, B., Holz, B., Iftner, A., Quint, W., et al. (2008). Comparison of the performance of different HPV genotyping methods for detecting genital HPV types. Journal of Medical Virology, 80(7), 1264-1274.

Kurman, R. J., Trimble, C. L., \& Shah, K. V. (1992). Human papillomavirus and the pathogenesis of vulvar carcinoma. Current Opinion in Obstetrics \& Gynecology, $4(4), 582-585$.

Levine, T. S., Rolfe, K. J., Crow, J., Styles, S., Perrett, C. W., Maclean, A. B., et al. (2001). The use of cytospin monolayer technique in the cytological diagnosis of vulval and anal disease. Cytopathology, 12(5), 297-305. 
Likes, W. M. (2009). Vulvar cancer in the wake of increasing incidence. The Nurse Practitioner, 34(2), 45-50.

Likes, W. M., \& Itano, J. (2003). Human papillomavirus and cervical cancer: not just a sexually transmitted disease. Clinical Journal of Oncology Nursing, 7(3), 271276.

Likes, W. M., Russell, C., \& Tillmanns, T. (2008). Women's experiences with vulvar intraepithelial neoplasia. Journal of Obstetric, Gynecologic, and Neonatal Nursing 37(6), 640-646.

Lin, M. C., Mutter, G. L., Trivijisilp, P., Boynton, K. A., Sun, D., \& Crum, C. P. (1998). Patterns of allelic loss (LOH) in vulvar squamous carcinomas and adjacent noninvasive epithelia. The American Journal of Pathology, 152(5), 1313-1318.

Longworth, M. S., \& Laimins, L. A. (2004). Pathogenesis of human papillomaviruses in differentiating epithelia. Microbiology and Molecular Biology Reviews, 68(2), 362-372.

Maclean, A. B. (2006). Vulval cancer: prevention and screening. Best Practice \& Research. Clinical Obstetrics \& Gynaecology, 20(2), 379-395.

Nauth, H. F. (1981). Definition of the terms "parakeratosis" and "dyskeratosis" from gynecocytological point of view (author's transl). Zeitschrift für Hautkrankheiten, 56(24), 1552-1563.

Nauth, H. F. (1986). Current vulvar diagnostic methods, with special reference to vulvar cytology. Journal of Reproductive Medicine, 31(9), 788-795.

Nauth, H. F., \& Boger, A. (1982). New aspects of vulvar cytology. Acta Cytologica, 26(1), 1-6.

Nauth, H. F., Neumann, G. K., \& Feilen, K. D. (1987). Structural and morphometric analysis of parakeratotic and dyskeratotic cells exfoliated from various vulvar lesions. Correlation with data from cervical cytology. Analytical and Quantitative Cytology and Histology, 9(3), 243-252.

Nonogaki, S., Wakamatsu, A., Longatto Filho, A., Pereira, S. M., Utagawa, M. L., Ferreira Alves, V. A., et al. (2004). Hybrid capture II and polymerase chain reaction for identifying HPV infections in samples collected in a new collection medium: a comparison. Acta Cytologica, 48(4), 514-520.

Pearl, W. S. (1999). A hierarchical outcomes approach to test assessment. Annals of Emergency Medicine, 33(1), 77-84.

Qu, W., Jiang, G., Cruz, Y., Chang, C. J., Ho, G. Y., Klein, R. S., et al. (1997). PCR detection of human papillomavirus: comparison between MY09/MY11 and GP5+/GP6+ primer systems. Journal of Clinical Microbiology, 35(6), 1304-1310. 
Scurry, J. (1999). Does lichen sclerosus play a central role in the pathogenesis of human papillomavirus negative vulvar squamous cell carcinoma? The itch-scratch-lichen sclerosus hypothesis. International Journal of Gynecological Cancer 9(2), 89-97.

Sherman, K. J., Daling, J. R., Chu, J., Weiss, N. S., Ashley, R. L., \& Corey, L. (1991). Genital warts, other sexually transmitted diseases, and vulvar cancer. Epidemiology, 2(4), 257-262.

Skapa, P., Zamecnik, J., Hamsikova, E., Salakova, M., Smahelova, J., Jandova, K., et al. (2007). Human papillomavirus (HPV) profiles of vulvar lesions: possible implications for the classification of vulvar squamous cell carcinoma precursors and for the efficacy of prophylactic HPV vaccination. The American Journal of Surgical Pathology, 31(12), 1834-1843.

Stroup, A. M., Harlan, L. C., \& Trimble, E. L. (2008). Demographic, clinical, and treatment trends among women diagnosed with vulvar cancer in the United States. Gynecologic Oncology, 108(3), 577-583.

Sturgeon, S. R., Brinton, L. A., Devesa, S. S., \& Kurman, R. J. (1992). In situ and invasive vulvar cancer incidence trends (1973 to 1987). American Journal of Obstetrics and Gynecology, 166(5), 1482-1485.

Thuis, Y. N., Campion, M., Fox, H., \& Hacker, N. F. (2000). Contemporary experience with the management of vulvar intraepithelial neoplasia. International Journal of Gynecological Cancer, 10(3), 223-227.

Vinokurova, S., Wentzensen, N., Einenkel, J., Klaes, R., Ziegert, C., Melsheimer, P., et al. (2005). Clonal history of papillomavirus-induced dysplasia in the female lower genital tract. Journal of the National Cancer Institute, 97(24), 1816-1821. 


\section{APPENDIX A. INSTITUTIONAL REVIEW BOARD APPROVAL}

$\begin{array}{lr}\text { THE UNIVERSITY OF TENNESSEE } \\ \text { Health Science Center } \\ \text { Anstitutional Review Board } \\ \text { August } 13,2007 & 910 \text { Madison Avenue, Suite } 600 \\ \text { Memphis, TN } 38163 \\ \text { Tel: (901) } 448-4824\end{array}$

Wendy Likes, DNSc

Nadeem Zafar, M.D.

College of Nursing

Department of Acute and Chronic Care

920 Madison, $\# 507 \mathrm{~N}$

CAMPUS MAIL

Dear Dr. Likes and Dr. Zafar:

The IRB has received your written acceptance of and/or responses dated August 9, 2007 and August 13, 2007 to the provisos outlined in my letters of August 3, 2007 and August 13, 2007 concerning the project entitled "Feasibility Study to Evaluate the Use of Liquid-Based Cytology with HPV Testing for PostTreatment Surveillance of Patients with Vulvar Intraepithelial Neoplasia" (IRB \#8712).

The IRB has reviewed these materials and determined that they do comply with proper consideration for the rights and welfare of human subjects and the regulatory requirements for the protection of human subjects. Therefore, this letter constitutes full approval by the IRB of your application and consent form dated August 13, 2007 (stamped IRB approved August 13, 2007). This study was approved for 12 months with an expiration date of July 31, 2008.

The IRB has also determined that the informed consent form, incorporating the authorization of subjects to use their protected health information in research, complies with the federal privacy regulations as specified in 45 CFR 160 and 45 CFR 164.

In the event that subjects are to be recruited using solicitation materials, such as brochures, posters, webbased advertisements, etc., these materials must receive prior approval of the IRB. Any revisions in the approved application must also be submitted to and approved by the IRB prior to implementation. In addition, you are responsible for reporting any unanticipated serious adverse events or other problems involving risks to subjects or others in the manner required by the local IRB policy.

Finally, re-approval of your project is required by the IRB in accord with the conditions specified above. You may not continue the research study beyond the time or other limits specified unless you obtain prior written approval of the IRB.

Sincerely,
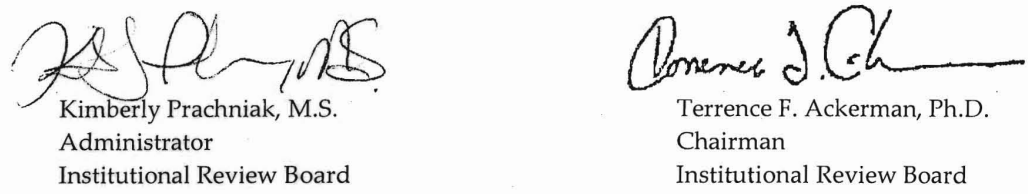


\title{
APPENDIX B. CONSENT FORM
}

Consent Form

Feasibility Study to Evaluate the Use of liquid-based cytology with HPV Testing for PostTreatment Surveillance of Patients with Vulvar Intraepithelial Neoplasia

\author{
Principal Investigator: \\ Wendy M. Likes, DNSc, APRN-Bc \\ \& \\ Nadeem Zafar, M.D. \\ 920 Madison Ave Suite 507N \\ Memphis, TN 38163 \\ Co Investigators: \\ Barbara DuBray-Benstein, Ph.D., SCT(ASCP) \\ Leonard I. Bloom, MPH, CT(ASCP)
}

\section{INTRODUCTION:}

You are being given the opportunity to participate in a research study. The purpose of the study is to determine whether tissue sampling, Human Papillomavirus (HPV) and p16 protein testing is feasible for monitoring precancerous tissue of the vulva, also called vulvar intraepithelial neoplasia (VIN). A neoplasia is an abnormal and uncontrolled growth of cells. Human papillomavirus (HPV) is a virus that can cause abnormal tissue growth and other changes to cells. Infection with HPV can increase the risk of developing cervical cancer. The vulva is the collection of external female genital organs including the clitoris, vaginal lips and opening to the vagina.

We are seeking a total of 75 participants. This study is being offered to women at UT Medical Group, Inc. OB/GYN Colposcopy Clinic located at the MED. A colposcopy is an examination of the vagina and cervix using a lighted magnifying instrument called a colposcope. The amount of time estimated for your participation in this study will not exceed one hour. The actual study procedure should lengthen your scheduled appointment by an additional 2-3 minutes.

\section{PROCEDURES TO BE FOLLOWED:}

If you agree to participate, you will be asked questions to see if you qualify for enrollment in the research study. Once it is identified that you qualify for the study, you will be asked to sign this consent form. Depending on your history, your consent to participate will allow us to collect cells from a healthy area of the vulva or one with possible VIN. If you have a prior history, you will still undergo the routine procedure for diagnosis of possible VIN areas, which is a vulvar punch biopsy, which will be performed by Dr. Likes, the principal investigator. Following the guidelines directed for routine care, $5 \%$ acetic acid (similar to table vinegar) will be applied to the vulva for 3-5 minutes and the vulva examined for any abnormal areas. If an area is identified as abnormal, the area will be outlined in black ink and a digital photo (not to include your face or anything identifiable) of the lesion (area of abnormal tissue) will be taken. The digital photographs will be taken in an effort to compare your visual findings with your laboratory results. The abnormal area will be numbed with lidocaine (a local anesthetic used to reduce pain) using a small gauge needle, after the area is cleaned with betadine (a topical antiseptic solution). Cells will then be collected by Dr. Likes using a small nylon brush soaked in a salt solution. Three samples will be collected one for microscopic viewing, one for HPV testing, and one for p16 protein testing. A tissue biopsy will then be taken, in cases where a lesion(s) is present, as is the standard with abnormal vulvar findings. This procedure will be repeated if it is clinically necessary to obtain more than one biopsy. The punch biopsy involves removal of a small, disk-shaped sample of tissue using a sharp, hollow instrument. The tissue is then examined under a microscope for any abnormalities. 
The anticipated duration of the one research visit will be no more than one hour. The total study duration is 12 months for enrollment of all 75 subjects. No further follow-up or contact will be needed, unless an abnormal finding occurs. If an abnormal result is found from any of your specimens i.e. biopsy, cytology, HPV, or p16 your healthcare provider will be notified so they can best determine your plan of care. No names or personally identifying information will be associated with any findings and the data will be reported as a whole without identifying you.

\section{RISKS ASSOCIATED WITH PARTICIPATION:}

In an effort to reduce the discomfort experienced with the research procedure, the area cleaned with betadine (if not allergic) will be anesthetized with injected lidocaine. Since the area will be numbed prior to collecting the cells for cytology and HPV testing, there should be minimal discomfort outside of the discomfort associated with the standard procedures performed for an abnormal vulvar finding. The risks associated with the cytologic sampling required for study procedure include possible irritation from the cytobrush and potential discomfort associated with photographs being taken. Risks associated with the standard practice of vulvar biopsy include pain, bleeding, and infection. You will be instructed by Dr. Likes not to rub area and to keep clean and dry for one week.

\section{BENEFITS ASSOCIATED WITH PARTICIPATION:}

You will receive no immediate benefits for participating in this study. However, there are potential benefits for you and other women in the future if cytology is found to be reliable and it is adopted into practice for VIN follow-up. If adopted into practice, cytology could provide an option for healthcare providers for screening VIN that is non-invasive and less painful for patients than the biopsy that is currently used for screening.

\section{ALTERNATIVES TO PARTICIPATION:}

You will receive your regular care in the clinic whether or not you participate in the study. If you choose not to participate, then you will not have a swab of the cells taken with the nylon brush, and you will not have photographs taken.

\section{CONFIDENTIALITY:}

Your research record and specimens will be labeled with a code number and a master key that links your name to the code number will be maintained in a separate and secure location. In the event that abnormal results are derived from your cytology, HPV and/or p16 testing, your code number will be linked to your name and results will be provided to you by Dr. Likes.

Your research record will be kept in a locked location only accessible to the principal investigator and the research staff. The digital photograph taken will include an index card placed next to the vulva that contains your code number in an effort to correlate clinical findings with the vulvar cytology results. A copy of your consent form will be included will be included in your medical record. As a result, third parties such as insurers or employers may have access to this information. You will not be identified in any presentations or publications based on the results of the research study. A code will be used to identify your cytology, HPV, and p16 specimen and results.

Under federal privacy regulations, you have the right to determine who has access to your personal health information (called "protected health information" or PHI). PHI collected in this study may include your medical history, the results of physical exams, lab tests, $x$-ray exams, and other diagnostic and treatment procedures, as well as basic demographics information. By signing this consent form, you are authorizing the researchers at the University of Tennessee and UT Medical Group, Inc. to have access to your PHI collected in this study and to receive your PHI from your physician and/or facilities where you have received health care. The Institutional Review Board (IRB) at the University of Tennessee Health Science Center may review your PHI as part of its responsibility to protect the rights and welfare of research subjects. Your PHI will not be used or disclosed to any other person or entity, except as required 
by law, or for authorized oversight of this research study by other regulatory agencies, or for other research for which the use and disclosure of your PHI has been approved by the IRB. Your PHI will be used only for the research purposes described in the Introduction of this consent form. Your PHI will be used until the study is completed.

You may cancel this authorization in writing at any time by contacting the principal investigator listed on the first page of the consent form. If you cancel the authorization, continued use of your PHI is permitted if it was obtained before the cancellation and its use is necessary in completing the research. However, $\mathrm{PHI}$ collected after your cancellation may not be used in the study. If you refuse to provide this authorization, you will not be able to participate in the research study. If you cancel the authorization, then you will be withdrawn from the study. Finally, the federal regulations allow you to obtain access to your PHI collected or used in this study.

\section{COMPENSATION AND TREATMENT FOR INIURY:}

I understand that I am not waiving any legal rights or releasing the

Health Science Center (UTHSC), its agents, UT Medical Group, Inc, ana ine ived rrom namury for negligence. I also understand that, in the event of physical injury resulting from research procedures, UTHSC, UT Medical Group, Inc., and The Med do not have funds budgeted for compensation either for lost wages or for medical treatment. Therefore, UTHSC, UT Medical Group, Inc., and The Med do not provide for treatment or reimbursement for such injuries. In the case of a research-related injury, Dr. Likes, the principal investigator will provide any additional care required for the treatment of the research-related injury. I and/or my insurance carrier will be responsible for the costs associated with any medical treatment for a researchrelated injury.

\section{QUESTIONS:}

You may contact the Principal Investigator listed on the front of this consent form with any questions about the research study. You may contact Terrence Ackerman, UTHSC IRB

(Institutional Review Board) Chairman at 901-448-4824 if you have questions about your rights as a participant in this study or your rights as a research subject. You may contact the Principal Investigator, Dr. Wendy Likes, at 901-355-4289 in the event of a research related injury 24 hours a day/ 7 days a week.

\section{PAYMENT FOR PARTICIPATION:}

There will not be any payment for participation in this study.

\section{COSTS OF PARTICIPATION:} There will not be any additional costs incurred by you as a result of participation in this
research study. Any cost associated with your participation in this study will be covered by the grant used to fund this study.

\section{PREMATURE TERMINATION:}

It is at the Principal Investigators discretion to terminate your participation in this research study. The only circumstance that would require premature termination from this study would be if not enough cells are seen during the cytology exam.

\section{VOLUNTARY PARTICIPATION:}

Your participation is voluntary and refusal to participate will involve no penalty or loss of benefits to which you are entitled. You may discontinue participation at any time without penalty or loss of benefits to which you are otherwise entitled.

13. CONSENT OF SUBIECT:

I have read or have had read to me the description of the research study as outlined above. The investigator or her representative has explained the study to me and has answered all of the 
questions I have at this time. I have been told of the potential risks, discomforts, side effects and adverse reactions as well as the possible benefits (if any) of the study.

I freely volunteer to participate in the study. I understand that I do not have to take part in this study and that my refusal to participate will involve no penalty or loss of rights to which I am entitled. I further understand that I am free to later withdraw my consent and discontinue participation in this study at any time. I understand that refusing to participate or later withdrawing from the study will not adversely affect my subsequent medical care.

I will receive a copy of the consent form.

Signature of Subject

Signature of Person Conducting Consent Interview

Signature of Witness

Signature of Principal Investigator

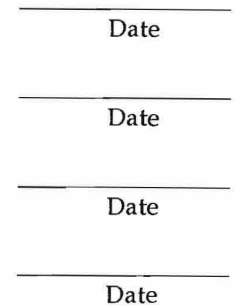

Date 


\section{VITA}

Wendy Likes was born November 8, 1972 in Columbus, Mississippi. She was awarded an Associate Degree from Arkansas State University and a Bachelor of Science in Nursing from The University of Memphis in Memphis, Tennessee, in 1994 and 1997, respectively. She graduated from The University of Tennessee Health Science Center, in 1999 with a Master of Science in Nursing as a Family Nurse Practitioner. In 2003, she was awarded a Doctor of Nursing Science Degree from The University of Tennessee Health Science Center.

Dr. Likes was certified as a Family Nurse Practitioner by ANCC in 1998. She completed a colposcopy mentorship program through the American Society of Colposcopy and Cervical Pathology and serves as a mentor for others through this organization. She is actively involved in many national and international organizations, serving on multiple committees, and was one of the first nurses inducted as a Fellow in the International Society for the Study of Vulvovaginal Disease. She has published several manuscripts as a primary and secondary author in the following journals: Journal of Sex and Marital Therapy, Gynecologic Oncology, Journal of Lower Genital Tract Disease, Journal of Reproductive Medicine, and The Nurse Practitioner. She has presented at several national and international conferences. She was awarded a K23 grant from the National Institute of Nursing Research in 2007 on Predictors of Quality of Life Following Vulvar Excision for Vulvar Intraepithelial Neoplasia and has built a program of research in sexuality and women's health with a focus on pre-invasive gynecologic disease.

Wendy Likes will receive a Doctor of Philosophy in Nursing degree from The University of Tennessee Health Science Center in May, 2009. 